\title{
Extremum-seeking control for optimization of time-varying steady-state responses of nonlinear systems
}

Citation for published version (APA):

Hazeleger, L., Haring, M., \& van de Wouw, N. (2020). Extremum-seeking control for optimization of time-varying steady-state responses of nonlinear systems. Automatica, 119, [109068].

https://doi.org/10.1016/j.automatica.2020.109068

\section{Document license:}

TAVERNE

DOI:

10.1016/j.automatica.2020.109068

Document status and date:

Published: 01/09/2020

Document Version:

Publisher's PDF, also known as Version of Record (includes final page, issue and volume numbers)

\section{Please check the document version of this publication:}

- A submitted manuscript is the version of the article upon submission and before peer-review. There can be important differences between the submitted version and the official published version of record. People interested in the research are advised to contact the author for the final version of the publication, or visit the $\mathrm{DOI}$ to the publisher's website.

- The final author version and the galley proof are versions of the publication after peer review.

- The final published version features the final layout of the paper including the volume, issue and page numbers.

Link to publication

\section{General rights}

Copyright and moral rights for the publications made accessible in the public portal are retained by the authors and/or other copyright owners and it is a condition of accessing publications that users recognise and abide by the legal requirements associated with these rights.

- Users may download and print one copy of any publication from the public portal for the purpose of private study or research.

- You may not further distribute the material or use it for any profit-making activity or commercial gain

- You may freely distribute the URL identifying the publication in the public portal.

If the publication is distributed under the terms of Article 25fa of the Dutch Copyright Act, indicated by the "Taverne" license above, please follow below link for the End User Agreement:

www.tue.nl/taverne

Take down policy

If you believe that this document breaches copyright please contact us at:

openaccess@tue.nl

providing details and we will investigate your claim. 


\title{
Extremum-seeking control for optimization of time-varying steady-state responses of nonlinear systems
}

\author{
Leroy Hazeleger ${ }^{\text {a,* }}$, Mark Haring ${ }^{\text {b }}$, Nathan van de Wouw ${ }^{\text {a,c }}$ \\ a Eindhoven University of Technology, Department of Mechanical Engineering, Eindhoven 5600 MB, The Netherlands \\ b SINTEF Digital, Mathematics, and Cybernetics, Trondheim, Norway \\ ${ }^{c}$ University of Minnesota, Department of Civil, Environmental and Geo-Engineering, Minneapolis, MN 55455, USA
}

\section{A R T I C L E I N F O}

\section{Article history:}

Received 11 July 2018

Received in revised form 24 March 2020

Accepted 4 May 2020

Available online 8 June 2020

\section{Keywords:}

Extremum-seeking control

Adaptive control

Performance optimization

Semi-global practical stability

Time-varying systems

\begin{abstract}
A B S T R A C T
Extremum-seeking control (ESC) is a useful tool for the steady-state performance optimization of plants for which limited knowledge about its dynamical behavior and disturbance situation is known. The case when the steady-state plant responses correspond to equilibrium solutions received a lot of attention. However, in many industrial applications plant performance is characterized by timevarying steady-state behavior. In those cases, no static parameter-to-steady-state performance map can be defined. In this work, we propose an ESC method that employs a so-called dynamic cost function to cope with time-varying steady-state responses of general nonlinear systems. We prove semi-global practical asymptotic stability of the closed-loop ESC scheme in the presence of bounded and time-varying external disturbances. Moreover, the working principle is illustrated by means of the real-time performance optimal tuning of a nonlinear control strategy for a motion control application.
\end{abstract} (C) 2020 Elsevier Ltd. All rights reserved.

\section{Introduction}

In most of the work on extremum-seeking control (ESC), the general requirement for optimizing a stable or stabilized plant is the existence of a (unknown) time-invariant parameter-tosteady-state performance map, i.e., a static input-output relation between tunable plant parameters and the steady-state plant performance, the extremum of which corresponds to the optimal steady-state (equilibrium) plant performance (Dürr, Krstić, Scheinker, \& Ebenbauer, 2017; Guay \& Dochain, 2015; Krstić \& Wang, 2000; Nešić, Tan, Moase, \& Manzie, 2010; Tan, Nešić, \& Mareels, 2006; Teel \& Popović, 2001). In those references, the requirements for the existence of such an input-output relation have been formalized in terms of stability properties for dynamical systems that admit equilibria solutions in steady-state. Even in the presence of (high-frequency) measurement noise, in, e.g., Fu and Özgüner (2011), Haring (2016), Haring and Johansen (2018), Stanković and Stipanović (2010), Tan, Moase, Manzie, Nešić, and Mareels (2010) and Zhang and Ordóñez (2009), ESC in the equilibrium setting to a neighborhood of the optimum

\footnotetext{
The material in this paper was partially presented at the 2018 American Control Conference, June 27-29, 2018, Milwaukee, WI, USA. This paper was recommended for publication in revised form by Associate Editor Björn S. Rüffer under the direction of Editor Daniel Liberzon.

* Corresponding author.

E-mail addresses: 1.hazeleger@tue.nl (L. Hazeleger), mark.haring@sintef.no (M. Haring), n.v.d.wouw@tue.nl (N. van de Wouw).
}

can be achieved, the size of which is often dependent on the noise level. However, in many (industrial) applications a static input-output relation does not exist because plant performance is generally related to generic, time-varying steady-state plant behavior. This time-varying plant behavior emerges, for example, in reference tracking or disturbance attenuation problems, which are encountered, for example, in industrial motion systems, such as, pick-and-place systems (van Loon, Hunnekens, Heemels, van de Wouw, \& Nijmeijer, 2016), electron microscopes, and wafer scanning systems (Heertjes \& Nijmeijer, 2012; Heertjes, Schuurbiers, \& Nijmeijer, 2009). Indeed, optimal steadystate plant performance and the corresponding optimal tuning are heavily dependent on the (time-varying) disturbance situation and reference trajectory at hand. It is essential to consider these generically time-varying disturbances and the resulting tobe-optimized performance in terms of generically time-varying steady-state responses as an integral part in the problem formulation of performance optimization of time-varying steady-state plant behavior.

In Guay, Dochain, Perrier, and Hudon (2007), Haring, van de Wouw, and Nešić (2013), and Wang and Krstić (2000), ESC approaches have been proposed to deal with time-varying, periodic steady-state behavior. Key in the proposed approaches is the design of a cost function that defines a constant performance measure in terms of the periodic steady-state response. In Wang and Krstić (2000), a so-called detector is proposed that is able to capture the amplitude of sinusoidal-like limit cycle responses, 
and has been applied, e.g., in Antonello, Oboe, Prandi, and Biganzoli (2009) and Bolder et al. (2012). In Guay et al. (2007), a class of differentially flat periodic nonlinear systems is considered. By exploiting flatness and explicit plant knowledge, a period of the periodic steady-state plant output and the corresponding steady-state plant performance are computed. In Höffner, Hudon, and Guay (2007), a similar approach is pursued for a class of periodic Hamiltonian systems. In Haring et al. (2013), general nonlinear plants with arbitrary periodic steady-state responses with known period time are considered. A cost functional based on an $\mathcal{L}^{p}$-norm, evaluated over the periodic time interval, is employed that links periodic steady-state responses to a timeinvariant performance measure. In Hunnekens, Di Dino, van de Wouw, van Dijk, and Nijmeijer (2015), this method was experimentally demonstrated for the adaptive design of variable-gain controllers for a motion control application. In many (industrial) applications, however, the steady-state response characterizing system performance is generically time-varying, and periodicity of the steady-state response is not evident due to the fact that responses can be induced by complex time-varying (non-periodic) disturbances and reference signals. In such generic cases, a static parameter-to-steady-state performance map may not be readily defined as in the periodic cases in Haring et al. (2013), Hunnekens et al. (2015), and Wang and Krstić (2000).

Other works that involve time-varying system behavior are (Scheinker \& Krstić, 2013; Scheinker \& Scheinker, 2016). In here, ESC is utilized as feedback control, able to, on the one hand, control unstable and time-varying input-affine systems, and on the other hand, optimize steady-state equilibria in the presence of noise. We must emphasize that, in our work, we consider the problem of optimizing time-varying steady-state system responses, which is a different problem from the one considered in Scheinker and Scheinker (2016) and Scheinker and Krstić (2013). Moreover, we remark that the methods in Scheinker and Scheinker (2016) and Scheinker and Krstić (2013) rely on high dither frequencies relative to the time-varying system dynamics, while our proposed method can employ small dither frequencies relative to the time-varying system dynamics, even in the presence of high frequency disturbances. In addition, the class of systems considered in our work is larger.

ESC for slowly time-varying performance maps is considered in, e.g., Cao, Dürr, Ebenbauer, Allgöwer, and Gao (2017), Fu and Özgüner (2011), Grushkovskaya, Dürr, Ebenbauer, and Zuyev (2017), Rušiti, Evangelisti, Oliveira, Gerdts, and Krstić (2019) and Sahneh, Hu, and Xie (2012). In here, optimal plant performance is obtained by tracking optimal time-varying plant parameters. We remark that this problem setting is different from the one considered in this work. We consider a time-varying steadystate problem setting in the spirit of Haring et al. (2013), Hunnekens et al. (2015) and Wang and Krstić (2000), although more general in terms of the time-varying nature of the disturbances. We propose an ESC method that seeks constant plant parameter settings that optimize steady-state plant performance in terms of time-varying steady-state system responses.

Preliminary results of this work were published in Hazeleger, Haring, and van de Wouw (2018). In particular, Hazeleger et al. (2018) present only a local stability result, while the proof was omitted. The stability analysis result that we present here is much stronger than the local stability result in Hazeleger et al. (2018). Moreover, we provide the full proof with all details and derivations. In addition, the ESC problem formulation presented in this work generalizes the work in Haring and Johansen (2018) in the sense that, in this work, generically time-varying disturbances and resulting to-be-optimized performance characterized in terms of generically time-varying steady-state responses are considered an integral part of the ESC problem formulation. In general, this extends the class of problems from those involving systems admitting equilibria solutions, as mostly considered in ESC literature and also in Haring and Johansen (2018), or periodically time-varying steady-state responses in Guay et al. (2007), Haring et al. (2013) and Wang and Krstić (2000), to those involving systems exhibiting generically time-varying steadystate responses.

The main contribution of this work can be summarized as follows. First, we propose an ESC approach for the optimization of generic, time-varying steady-state responses of general nonlinear systems. A so-called dynamic cost function in terms of generically time-varying output responses is developed, allowing for the characterization of a time-invariant parameterto-steady-state performance map. Second, we prove semi-global practical asymptotic stability of the closed-loop ESC scheme that includes general nonlinear systems that are subject to bounded and time-varying external disturbances. The region to which the ESC scheme converges can be made arbitrarily small, even in the presence of generically time-varying, bounded disturbances. Third, the working principle of the proposed ESC method is illustrated by means of the real-time performance optimal tuning of a nonlinear control strategy for a motion control application within an industrial wafer scanner exhibiting time-varying steady-state responses.

The paper is organized as follows. Section 2 presents the problem formulation. Section 3 gives the extremum-seeking controller. In Section 4, the semi-global practical asymptotic stability result is stated and proven. In Section 5, the industrial case study is provided. Section 6 closes with conclusions.

\section{Extremum-seeking control problem formulation for time- varying system responses}

Consider the following generic description of a multiple-inputmultiple-output nonlinear system:

$\Sigma_{p}:\left\{\begin{array}{l}\dot{\mathbf{x}}(t)=\mathbf{f}(\mathbf{x}(t), \mathbf{u}(t), \mathbf{w}(t)) \\ \mathbf{e}(t)=\mathbf{g}(\mathbf{x}(t), \mathbf{u}(t), \mathbf{w}(t))\end{array}\right.$

where $\mathbf{x} \in \mathbb{R}^{n_{\mathbf{x}}}$ denotes the state of the system, $\mathbf{u} \in \mathbb{R}^{n_{\mathbf{u}}}$ denotes the input of the system, $\mathbf{e} \in \mathbb{R}^{n_{\mathbf{e}}}$ denotes the output of the system, $\mathbf{w} \in \mathbb{R}^{n_{\mathbf{w}}}$ are disturbances, and $t \in \mathbb{R}$ is time. In the context of ESC, $\Sigma_{p}$ represents the system to be optimized, where the input $\mathbf{u}$ can be regarded as a vector of tunable system parameters, the output e can be regarded as a vector of measured performance variables, and $\mathbf{w}$ are piecewise continuous (timevarying) disturbances, defined and bounded on $t \in \mathbb{R}$. We denote this class of disturbances by $\overline{\mathbb{P C}}_{\mathbf{w}}$, and define the following set of disturbances $\mathcal{W}:=\left\{\mathbf{w} \in \overline{\mathbb{P C}}_{\mathbf{w}}:\|\mathbf{w}(t)\| \leq \rho_{\mathbf{w}} \forall t \in \mathbb{R}\right\}$, with $\rho_{\mathbf{w}} \in \mathbb{R}_{>0}$. Although the functions $\mathbf{f}$ and $\mathbf{g}$ in (1) are considered unknown, we adopt the following assumption.

Assumption 1. The functions $\mathbf{f}: \mathbb{R}^{n_{\mathbf{x}}} \times \mathbb{R}^{n_{\mathbf{u}}} \times \mathbb{R}^{n_{\mathbf{w}}} \rightarrow \mathbb{R}^{n_{\mathbf{x}}}$ and $\mathbf{g}: \mathbb{R}^{n_{\mathbf{x}}} \times \mathbb{R}^{n_{\mathbf{u}}} \times \mathbb{R}^{n_{\mathbf{w}}} \rightarrow \mathbb{R}^{n_{\mathbf{e}}}$ are twice continuously differentiable in $\mathbf{x}$ and $\mathbf{u}$ and continuous in $\mathbf{w}$. Moreover, given any disturbance $\mathbf{w} \in \mathcal{W}$, there exist constants $L_{\mathbf{f x}}, L_{\mathbf{f u}}, L_{\mathbf{g x}}, L_{\mathbf{g u}} \in \mathbb{R}_{>0}$ such that

$\left\|\frac{\partial \mathbf{f}}{\partial \mathbf{x}}(\mathbf{x}, \mathbf{u}, \mathbf{w})\right\| \leq L_{\mathbf{f x}}, \quad\left\|\frac{\partial \mathbf{f}}{\partial \mathbf{u}}(\mathbf{x}, \mathbf{u}, \mathbf{w})\right\| \leq L_{\mathbf{f u}}$,
$\left\|\frac{\partial \mathbf{g}}{\partial \mathbf{x}}(\mathbf{x}, \mathbf{u}, \mathbf{w})\right\| \leq L_{\mathbf{g x}}, \quad\left\|\frac{\partial \mathbf{g}}{\partial \mathbf{u}}(\mathbf{x}, \mathbf{u}, \mathbf{w})\right\| \leq L_{\mathbf{g u}}$,

for all $\mathbf{x} \in \mathbb{R}^{n_{\mathbf{x}}}$, and all $\mathbf{u} \in \mathbb{R}^{n_{\mathbf{u}}}$

To define the classes of systems considered in the ESC problem setting, we first give definitions of so-called convergent systems which we have adopted from Pavlov, van de Wouw, and Nijmeijer (2006). 
Definition 2 (Pavlov et al., 2006, Section 2.2.1). A system of the form $\dot{\mathbf{x}}=\mathbf{F}(\mathbf{x}, t)$, where $\mathbf{x} \in \mathbb{R}^{n_{\mathbf{x}}}, t \in \mathbb{R}$, and $\mathbf{F}(\mathbf{x}, t)$ locally Lipschitz in $\mathbf{x}$ and piecewise continuous in $t$, is said to be

- convergent in a set $\mathcal{X} \subset \mathbb{R}^{n_{\mathbf{x}}}$ if there exists a solution $\overline{\mathbf{x}}(t)$, called the steady-state solution, satisfying the following conditions:

(i) $\overline{\mathbf{x}}(t)$ is defined and bounded for all $t \in \mathbb{R}$,

(ii) $\overline{\mathbf{x}}(t)$ is asymptotically stable in $\mathcal{X}$.

- uniformly convergent in $\mathcal{X}$ if it is convergent in $\mathcal{X}$ and $\overline{\mathbf{x}}(t)$ is uniformly asymptotically stable in $\mathcal{X}$.

- exponentially convergent in $\mathcal{X}$ if it is convergent in $\mathcal{X}$ and $\overline{\mathbf{x}}(t)$ is exponentially stable in $\mathcal{X}$.

- uniformly exponentially convergent in $\mathcal{X}$ if it is convergent in $\mathcal{X}$ and $\overline{\mathbf{x}}(t)$ is uniformly exponentially stable in $\mathcal{X}$.

If the system is (uniformly, exponentially) convergent in $\mathcal{X}=\mathbb{R}^{n_{\mathbf{x}}}$, then it is called globally (uniformly, exponentially) convergent.

Definition 3 (Pavlov et al., 2006, Section 2.2.2). A system of the form $\dot{\mathbf{x}}=\mathbf{F}(\mathbf{x}, \mathbf{v})$, with state $\mathbf{x} \in \mathbb{R}^{n_{\mathbf{x}}}$, input $\mathbf{v} \in \mathbb{R}^{n_{\mathbf{v}}}$, and $\mathbf{F}(\mathbf{x}, \mathbf{v})$ locally Lipschitz in $\mathbf{x}$ and continuous in $\mathbf{v}$, is said to be (uniformly, exponentially) convergent in $\mathcal{X} \subset \mathbb{R}^{n_{\mathbf{x}}}$ for a class of inputs $\mathcal{V} \subset \overline{\mathbb{P C}}_{\mathbf{v}}$ if it is (uniformly, exponentially) convergent in $\mathcal{X}$ for every input $\mathbf{v}(\cdot) \in \mathcal{V}$. In order to emphasize the dependency of the steady-state solution on the input $\mathbf{v}(t)$, it is denoted by $\overline{\mathbf{x}}_{\mathbf{v}}(t)$. Then, there exists a $\mathcal{K} \mathcal{L}$-function $\beta(r, s)$ such that any solution $\mathbf{x}(t)$ of the system satisfies

$$
\left\|\mathbf{x}(t)-\overline{\mathbf{x}}_{\mathbf{v}}(t)\right\| \leq \beta\left(\left\|\mathbf{x}\left(t_{0}\right)-\overline{\mathbf{x}}_{\mathbf{v}}\left(t_{0}\right)\right\|, t-t_{0}\right) .
$$

Definition 4 (Pavlov et al., 2006, Section 2.2.2). A system of the form $\dot{\mathbf{x}}=\mathbf{F}(\mathbf{x}, \mathbf{v})$, with state $\mathbf{x} \in \mathbb{R}^{n_{\mathbf{x}}}$, input $\mathbf{v} \in \mathbb{R}^{n_{\mathbf{v}}}$, and $\mathbf{F}(\mathbf{x}, \mathbf{v})$ locally Lipschitz in $\mathbf{x}$ and continuous in $\mathbf{v}$, is said to be (exponentially) input-to-state convergent if it is globally uniformly (exponentially) convergent for the class of inputs $\overline{\mathbb{P C}}_{\mathbf{v}}$, and for every input $\mathbf{v}(\cdot) \in \overline{\mathbb{P C}}_{\mathbf{v}}$ the system is input-to-state stable (ISS) with respect to the steady-state solution $\overline{\mathbf{x}}_{\mathbf{v}}(t)$, i.e., there exist a $\mathcal{K} \mathcal{L}$-function $\beta(r, s)$ and a $\mathcal{K}_{\infty}$-function $\gamma(r)$ such that any solution $\mathbf{x}(t)$ of the system corresponding to some perturbed input $\hat{\mathbf{v}}(t):=\mathbf{v}(t)+\Delta \mathbf{v}(t)$ satisfies

$$
\begin{aligned}
\left\|\mathbf{x}(t)-\overline{\mathbf{x}}_{\mathbf{v}}(t)\right\| \leq & \beta\left(\left\|\mathbf{x}\left(t_{0}\right)-\overline{\mathbf{x}}_{\mathbf{v}}\left(t_{0}\right)\right\|, t-t_{0}\right) \\
& +\gamma\left(\sup _{t_{0} \leq \tau \leq t}\|\Delta \mathbf{v}(\tau)\|\right) .
\end{aligned}
$$

We adopt the following assumption on the system in (1).

Assumption 5. The nonlinear system $\Sigma_{p}$ in (1) is globally uniformly exponentially convergent for a class of disturbances $\mathbf{w}(\cdot) \in \mathcal{W}$, and for all constant inputs $\mathbf{u} \in \mathbb{R}^{n_{\mathbf{u}}}$, uniformly in $\mathbf{u}$. In addition, given a disturbance $\mathbf{w} \in \mathcal{W}$ the globally exponentially stable (GES) steady-state solution, which we denote by $\overline{\mathbf{x}}_{\mathbf{w}}(t, \mathbf{u})$, is twice continuously differentiable in $\mathbf{u}$ and satisfies

$\left\|\frac{\partial \overline{\mathbf{x}}_{\mathbf{w}}}{\partial \mathbf{u}}(t, \mathbf{u})\right\| \leq L_{\mathbf{x u}}$

for all $t \in \mathbb{R}$, all $\mathbf{u} \in \mathbb{R}^{n_{\mathbf{u}}}$, and some constant $L_{\mathbf{x u}} \in \mathbb{R}_{>0}$.

Remark 6. Assumption 5 is the time-varying analogue of the common assumption in the ESC literature on the system exhibiting globally exponentially stable equilibria (Krstić \& Wang, 2000; Tan et al., 2010, 2006). This assumption is not considered restrictive, as it is exactly this property that extends the needed stability property from the equilibria setting to the time-varying steady-state setting considered in this work. A similar assumption is imposed on the nonlinear plant in the periodic steady-state settings in Haring et al. (2013) and Wang and Krstić (2000). Moreover, in many (nonlinear) control problems, for example reference tracking, synchronization, observer design and output regulation problems, the convergent system property that all solutions of a closed-loop system converges to the same steadystate solution and thus "forget" their initial condition plays an important role (Pavlov \& van de Wouw, 2016; Pavlov et al., 2006). In addition, the exponential property is immediate for asymptotically stable linear time-invariant systems with inputs.

Remark 7. A different notation for the steady-state solution can be $\overline{\mathbf{x}}_{\mathbf{w}, \mathbf{u}}(t)$, which agrees with Definition 3 and $\mathbf{v}^{\top}:=\left[\mathbf{u}^{\top}, \mathbf{w}^{\top}\right]$. Here, we opt for the notation $\overline{\mathbf{x}}_{\mathbf{w}}(t, \mathbf{u})$, on the one hand, to emphasize the dependency on time-varying disturbances $\mathbf{w}(t)$, and on the other hand, to be able to explicitly distinguish between the dependency on either constant inputs $\mathbf{u}$ or time-varying inputs $\mathbf{u}(t)$. This will prove instrumental in the scope of the stability analysis of the ESC scheme proposed later.

Given Assumption 5, for constant inputs $\mathbf{u} \in \mathbb{R}^{n_{\mathbf{u}}}$, there exists a unique, time-varying steady-state output of the system $\Sigma_{p}$ in (1), denoted by $\overline{\mathbf{e}}_{\mathbf{w}}(t, \mathbf{u})$, which is given by

$\overline{\mathbf{e}}_{\mathbf{w}}(t, \mathbf{u})=\mathbf{g}\left(\overline{\mathbf{x}}_{\mathbf{w}}(t, \mathbf{u}), \mathbf{u}, \mathbf{w}(t)\right)$.

We aim to find constant inputs $\mathbf{u}$ that optimize the steady-state performance of the system in (1). Common practice in the ESC literature is to define a cost function in terms of the system responses and inputs that quantifies the performance of interest for the system under study. For example, consider performance measures of the following form and adopted from (Haring et al., 2013):

$$
\begin{aligned}
L_{p}(t, \mathbf{e}(t)) & :=\frac{1}{T} \int_{t-T}^{t}\|\mathbf{e}(\tau)\|^{p} d \tau, \\
L_{\infty}(t, \mathbf{e}(t)) & :=\max _{\tau \in[t-T, t]}\|\mathbf{e}(\tau)\|,
\end{aligned}
$$

with $p \in[1, \infty)$, where $T \in \mathbb{R}_{>0}$ is a known performance relevant time-interval. In cases where, for constant inputs $\mathbf{u}$, the steady-state plant outputs $\overline{\mathbf{e}}_{\mathbf{w}}$ in (6) are constant or periodic with period time $T$, the steady-state output of the cost functions in (7) is constant as well. Having a constant steady-state output of the cost function for constant inputs $\mathbf{u}$ is one of the basic requirements in the ESC literature to be able to accomplish extremum seeking (Haring et al., 2013; Krstić \& Wang, 2000; Tan et al., 2006). However, in many (industrial) applications this requirement is not met, as the steady-state plant outputs $\overline{\mathbf{e}}_{\mathbf{w}}$ that characterize system performance are generically time-varying in nature (also for constant $\mathbf{u}$ ). In addition, periodicity of the steadystate plant outputs $\overline{\mathbf{e}}_{\mathbf{w}}$ is not evident due to the fact that system responses can be induced by complex time-varying, possibly nonperiodic disturbances and reference trajectories. In those cases, often the neighborhood to which the ESC scheme converges cannot be made arbitrarily small, thereby limiting the achievable performance gain.

To deal with the time-varying nature of the system responses, consider the series connection of the system $\Sigma_{p}$ as in (1), a cost function $Z$ of the form

$y(t)=Z(\mathbf{e}(t), \mathbf{u}(t))$,

where $y \in \mathbb{R}$, and additionally a user-defined filter, denoted by $\Sigma_{f}$, which has the following general form

$\Sigma_{f}:\left\{\begin{aligned} \dot{\mathbf{z}}(t) & =\alpha_{\mathbf{z}} \mathbf{h}(\mathbf{z}(t), y(t)) \\ l(t) & =k(\mathbf{z}(t)),\end{aligned}\right.$

where $\alpha_{\mathbf{z}} \in \mathbb{R}_{>0}$ is a tuning parameter, $\mathbf{z} \in \mathbb{R}^{n_{\mathbf{z}}}$ is the state of the filter, $y \in \mathbb{R}$ is the input of the filter defined by (8), and 
$l \in \mathbb{R}$ is the output of the filter. We call the series connection of the cost function $Z$ and the filter $\Sigma_{f}$ the dynamic cost function. The function $Z: \mathbb{R}^{n_{\mathbf{e}}} \times \mathbb{R}^{n_{\mathbf{u}}} \rightarrow \mathbb{R}$ is designed to be twice continuously differentiable with respect to both arguments. Moreover, we choose $Z$ in such a way that there exist constants $L_{Z \mathbf{e}}, L_{Z \mathbf{u}} \in \mathbb{R}_{>0}$ such that

$\left\|\frac{\partial^{2} Z}{\partial \mathbf{e} \partial \mathbf{e}^{\top}}(\mathbf{e}, \mathbf{u})\right\| \leq L_{Z \mathbf{e}},\left\|\frac{\partial^{2} Z}{\partial \mathbf{e} \partial \mathbf{u}^{\top}}(\mathbf{e}, \mathbf{u})\right\| \leq L_{Z \mathbf{u}}$,

for all $\mathbf{e} \in \mathbb{R}^{n_{\mathbf{e}}}$, and all $\mathbf{u} \in \mathbb{R}^{n_{\mathbf{u}}}$. Given a disturbance $\mathbf{w} \in \mathcal{W}$, for all constant inputs $\mathbf{u} \in \mathbb{R}^{n_{\mathbf{u}}}$, the steady-state output of $Z$ is denoted by $\bar{y}_{\mathbf{w}}(t, \mathbf{u})$ and reads

$\bar{y}_{\mathbf{w}}(t, \mathbf{u})=Z\left(\mathbf{g}\left(\overline{\mathbf{x}}_{\mathbf{w}}(t, \mathbf{u}), \mathbf{u}, \mathbf{w}(t)\right), \mathbf{u}\right)$.

The functions $\mathbf{h}: \mathbb{R}^{n_{\mathbf{z}}} \times \mathbb{R} \rightarrow \mathbb{R}^{n_{\mathbf{z}}}$ and $k: \mathbb{R}^{n_{\mathbf{z}}} \rightarrow \mathbb{R}$ in (9) are designed to be twice continuously differentiable with respect to all arguments. Moreover, we design $\Sigma_{f}$ in such a way that there exist constants $L_{\mathbf{h z}}, L_{\mathbf{h} y}, L_{k} \in \mathbb{R}_{>0}$ such that

$\left\|\frac{\partial \mathbf{h}}{\partial \mathbf{z}}(\mathbf{z}, y)\right\| \leq L_{\mathbf{h} \mathbf{z}},\left\|\frac{\partial \mathbf{h}}{\partial y}(\mathbf{z}, y)\right\| \leq L_{\mathbf{h} y},\left\|\frac{d k}{d \mathbf{z}}(\mathbf{z})\right\| \leq L_{k}$,

for all $\mathbf{z} \in \mathbb{R}^{n_{\mathbf{z}}}$, and all $y \in \mathbb{R}$. In addition, we design the dynamic cost function to satisfy the following property.

Property 8. The dynamic cost function consisting of the cascade of $Z$ and $\Sigma_{f}$, given by (8) and (9), respectively, is exponentially inputto-state convergent for all constant inputs $\mathbf{u} \in \mathbb{R}^{n_{\mathbf{u}}}$ and all $\alpha_{\mathbf{z}} \in \mathbb{R}_{>0}$, uniformly in $\mathbf{u}$.

Intuitively, the filter $\Sigma_{f}$ is introduced to act as an averaging operator on $y(t)$, utilized to quantify performance of the system similar to the use of exponentially weighting filters (Antonello et al., 2009; Wang \& Krstić, 2000). By tuning $\alpha_{\mathbf{z}}$ small the solution $\mathbf{z}(t)$ will vary "slowly" in time, i.e., the output of the filter $l(t)$ will be quasi-constant and determined predominantly by the average of $y(t)$. By properly designing cost function $Z$ and tuning $\alpha_{\mathbf{z}}$ in (9) small, the output of the dynamic cost function $l(t)$ is quasiconstant and reflects the performance of the system, while being characterized by the time-varying system response $\mathbf{e}(t)$. By subsequently minimizing $l(t)$ using ESC, we optimize the time-varying system response $\mathbf{e}(t)$.

Remark 9. In the scope of the proposed approach, a sufficiently slow filter $\Sigma_{f}$ is able to approximate the time-average of $y(t)$ in real-time. This reveals the fact that the proposed approach introduces an additional time-scale to the extremum-seeking loop. That is, the filter $\Sigma_{f}$ must operate on a slower time-scale than the slowest one in $y(t)$, but fast enough for gradient estimation in ESC. Depending on the disturbance situation, this may lead to an increased convergence time of the ESC strategy. However, the introduction of the dynamic cost function, and the associated time scale, is essential to enable dealing with generic timevarying steady-state responses. Note that, also in cases where time-varying disturbances contain both low- and high-frequency behavior, our approach can still be used to achieve extremum seeking.

The series connection of the nonlinear system $\Sigma_{p}$ in (1) and the dynamic cost function is referred to as the extended plant $\Sigma$ and is schematically depicted in Fig. 1. The dynamics of the extended plant is given by

$\Sigma:\left\{\begin{array}{l}\dot{\mathbf{x}}(t)=\mathbf{f}(\mathbf{x}(t), \mathbf{u}(t), \mathbf{w}(t)) \\ \dot{\mathbf{z}}(t)=\alpha_{\mathbf{z}} \mathbf{h}(\mathbf{z}(t), Z(\mathbf{g}(\mathbf{x}(t), \mathbf{u}(t), \mathbf{w}(t)), \mathbf{u}(t))) \\ l(t)=k(\mathbf{z}(t)) .\end{array}\right.$

The following lemma deals with the series connection of a globally exponentially convergent system as in Definition 3 and an exponentially input-to-state convergent system as in Definition 4 and will prove instrumental in the scope of the stability analysis of the ESC scheme proposed later.

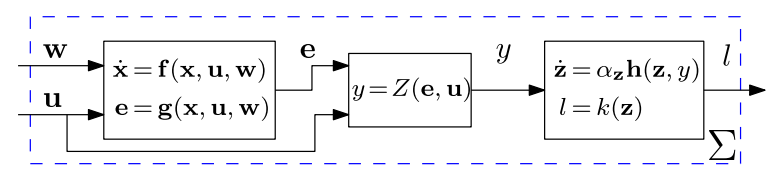

Fig. 1. The extended plant $\Sigma$, i.e., series connection of the nonlinear system $\Sigma_{p}$, the user-defined cost function $Z$, and the to-be-designed filter $\Sigma_{f}$.

Lemma 10. Consider the cascaded system

$\dot{\mathbf{x}}=\mathbf{F}(\mathbf{x}, \mathbf{v}), \quad \dot{\mathbf{z}}=\mathbf{G}(\mathbf{z}, \mathbf{x})$,

with $\mathbf{x} \in \mathbb{R}^{n_{\mathbf{x}}}, \mathbf{z} \in \mathbb{R}^{n_{\mathbf{z}}}, \mathbf{v} \in \mathbb{R}^{n_{\mathbf{v}}}$, and $\mathbf{F}$ and $\mathbf{G}$ locally Lipschitz in their first argument and continuous in their second argument. Consider inputs $\mathbf{v} \in \mathcal{V} \in \overline{\mathbb{P C}}_{\mathbf{v}}$. Suppose the $\mathbf{x}$-subsystem is globally uniformly exponentially convergent, and the $\mathbf{z}$-subsystem with $\mathbf{x}$ as input is exponentially input-to-state convergent. Then, the cascaded system in (14) is globally uniformly exponentially convergent.

Proof. The proof of Lemma 10 follows similar arguments as the proof of Property 2.27 in Pavlov et al. (2006, Section 2.2.3). Different from the result of Property 2.27 in Pavlov et al. (2006, Section 2.2.3) is the consideration of the series connection of a (globally uniformly exponentially) convergent system and an (exponentially) input-to-state convergent system, instead of the series connection of two (exponentially) input-to-state convergent subsystems. The resulting cascaded system is (globally uniformly exponentially) convergent, instead of (exponentially) input-to-state convergent.

Given Assumption 5 and Property 8, it follows from Lemma 10 that the extended plant $\Sigma$ in (13) is globally uniformly exponentially convergent for all constant inputs $\mathbf{u} \in \mathbb{R}^{n_{\mathbf{u}}}$, uniformly in $\mathbf{u}$. There exists a unique steady-state solution of $\Sigma_{f}$, induced by the extended plant, which is defined and bounded on $t \in \mathbb{R}$ and GES. We denote this steady-state solution by $\overline{\mathbf{z}}_{\mathbf{w}}\left(t, \mathbf{u}, \alpha_{\mathbf{z}}\right)$ to emphasize the dependency the time-varying disturbance $\mathbf{w}(t)$, constant inputs $\mathbf{u}$, and the tunable parameter $\alpha_{\mathbf{z}}$. For the steadystate solution of the extended plant $\Sigma$, we adopt the following assumption.

Assumption 11. Given a disturbance $\mathbf{w}(t) \in \mathcal{W}$, there exists a twice continuously differentiable function $\mathbf{q}_{\mathbf{w}}: \mathbb{R}^{n_{\mathbf{u}}} \rightarrow \mathbb{R}^{n_{\mathbf{z}}}$, referred to as the constant performance cost, such that

$\mathbf{q}_{\mathbf{w}}(\mathbf{u})=\lim _{\alpha_{\mathbf{z}} \rightarrow 0} \overline{\mathbf{z}}_{\mathbf{w}}\left(t, \mathbf{u}, \alpha_{\mathbf{z}}\right)$,

for all $t \in \mathbb{R}$, and all $\mathbf{u} \in \mathbb{R}^{n_{\mathbf{u}}}$. Moreover, there exist constants $\delta_{\mathbf{z} 1}, \delta_{\mathbf{z} 2} \in \mathbb{R}_{\geq 0}$, related to the disturbance $\mathbf{w}(t)$ and the extended plant $\Sigma$, such that the difference between the steady-state solution $\overline{\mathbf{z}}_{\mathbf{w}}\left(t, \mathbf{u}, \alpha_{\mathbf{z}}\right)$ and the function $\mathbf{q}_{\mathbf{w}}(\mathbf{u})$ satisfies

$\left\|\overline{\mathbf{z}}_{\mathbf{w}}\left(t, \mathbf{u}, \alpha_{\mathbf{z}}\right)-\mathbf{q}_{\mathbf{w}}(\mathbf{u})\right\| \leq \alpha_{\mathbf{z}}\left(\delta_{\mathbf{z} 1}+\delta_{\mathbf{z} 2}\left\|\mathbf{u}-\mathbf{u}_{\mathbf{w}}^{*}\right\|^{2}\right)$,

for all $t \in \mathbb{R}$, all $\mathbf{u} \in \mathbb{R}^{n_{\mathbf{u}}}$, and all $0<\alpha_{\mathbf{z}} \leq \epsilon_{\mathbf{z}}$ for some constant $\epsilon_{\mathbf{z}} \in \mathbb{R}_{>0}$, where $\mathbf{u}_{\mathbf{w}}^{*}$ denotes the optimal vector of tunable system parameters. In addition, $\bar{y}_{\mathbf{w}}(t, \mathbf{u})$ and $\overline{\mathbf{z}}_{\mathbf{w}}\left(t, \mathbf{u}, \alpha_{\mathbf{z}}\right)$ are twice continuously differentiable in $\mathbf{u}$. There exist constants $L_{y 1}, L_{y 2}, L_{\mathbf{q}}, L_{\mathbf{z} 1}, L_{\mathbf{z} 2} \in \mathbb{R}_{>0}$ such that

$\left\|\frac{\partial \bar{y}_{\mathbf{w}}(t, \mathbf{u})}{\partial \mathbf{u}}\right\| \leq L_{y 1}+L_{y 2}\left\|\mathbf{u}-\mathbf{u}_{\mathbf{w}}^{*}\right\|$

$\left\|\frac{d \mathbf{q}_{\mathbf{w}}}{d \mathbf{u}}(\mathbf{u})\right\| \leq L_{\mathbf{q}}\left\|\mathbf{u}-\mathbf{u}_{\mathbf{w}}^{*}\right\|$,

$\left\|\frac{\partial \overline{\mathbf{z}}_{\mathbf{w}}}{\partial \mathbf{u}}\left(t, \mathbf{u}, \alpha_{\mathbf{z}}\right)-\frac{d \mathbf{q}_{\mathbf{w}}}{d \mathbf{u}}(\mathbf{u})\right\| \leq \alpha_{\mathbf{z}}\left(L_{\mathbf{z} 1}+L_{\mathbf{z} 2}\left\|\mathbf{u}-\mathbf{u}_{\mathbf{w}}^{*}\right\|\right)$,

for all $t \in \mathbb{R}$, all $\mathbf{u} \in \mathbb{R}^{n_{\mathbf{u}}}$, all $0<\alpha_{\mathbf{z}} \leq \epsilon_{\mathbf{z}}$. 

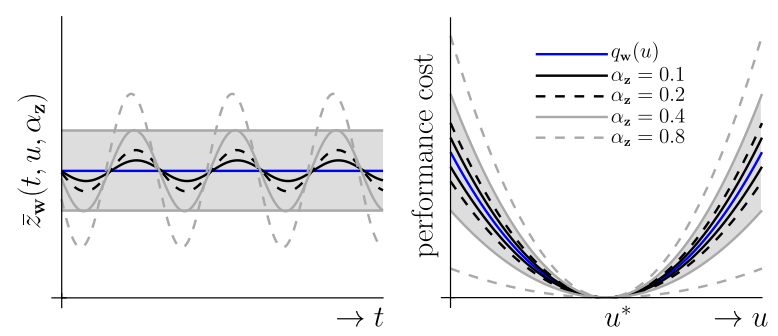

Fig. 2. A visualization of $z_{\mathbf{w}}\left(t, u, \alpha_{\mathbf{z}}\right)$ for some value of $u$ (left) and $q_{\mathbf{w}}(u)$ (right) for the example given in Remark 12. By tuning $\alpha_{\mathbf{z}}$ small, $z_{\mathbf{w}}\left(t, u, \alpha_{\mathbf{z}}\right)$ can be made arbitrarily close to $q_{\mathbf{w}}(u)$. The shaded area corresponds to the area in which $z_{\mathbf{w}}\left(t, u, \alpha_{\mathbf{z}}\right)$ lies when $\alpha_{\mathbf{z}}=0.4$.

Remark 12. Let us give an example of the working principles of the dynamic cost function, and provide intuition about Assumption 11. Consider, e.g., the time-varying steady-state output $\bar{e}_{\mathbf{w}}$ of (1) to be $\bar{e}_{\mathbf{w}}(t, u)=\left(u-u_{\mathbf{w}}^{*}\right) \sin (t)$ for which we want to minimize the amplitude, similar to the work in Wang and Krstić (2000). Here, we employ the following dynamic cost function, with cost function $Z: y(t)=e(t)^{2}$, and filter $\Sigma_{f}$ given by a firstorder low-pass filter $\dot{z}(t)=\alpha_{\mathbf{z}}(y(t)-z(t)), l(t)=z(t)$ with $\alpha_{\mathbf{z}}$ the cut-off frequency. The steady-state solution $\bar{z}_{\mathbf{w}}\left(t, u, \alpha_{\mathbf{z}}\right)$ then reads

$\bar{z}_{\mathbf{w}}\left(t, u, \alpha_{\mathbf{z}}\right)=\frac{1}{2}\left(u-u_{\mathbf{w}}^{*}\right)^{2}\left(1-\alpha_{\mathbf{z}} \frac{\alpha_{\mathbf{z}} \cos (2 t)+2 \sin (2 t)}{\alpha_{\mathbf{z}}^{2}+4}\right)$.

From (15), we obtain the constant performance cost as

$q_{\mathbf{w}}(u)=\lim _{\alpha_{\mathbf{z}} \rightarrow 0} \bar{z}_{\mathbf{w}}\left(t, u, \alpha_{\mathbf{z}}\right)=\frac{1}{2}\left(u-u_{\mathbf{w}}^{*}\right)^{2}$.

The function $q_{\mathbf{w}}(u)$ is twice continuously differentiable. Moreover, it follows that the difference between $\bar{z}_{\mathbf{w}}\left(t, u, \alpha_{\mathbf{z}}\right)$ and $q_{\mathbf{w}}$ can be bounded as follows

$\left|\bar{z}_{\mathbf{w}}\left(t, u, \alpha_{\mathbf{z}}\right)-q_{\mathbf{w}}(u)\right| \leq \frac{1}{2} \alpha_{\mathbf{z}}\left|u-u_{\mathbf{w}}^{*}\right|^{2}$.

Clearly, the inequality in (16) is satisfied with $\delta_{\mathbf{z} 1}=0$ and $\delta_{\mathbf{z} 2}=$ $\frac{1}{2}$, and tells us that the bound on the difference between the timevarying steady-state solution and the constant performance cost of the extended plant $\Sigma$ is tunable by $\alpha_{\mathbf{z}}$. By tuning $\alpha_{\mathbf{z}}$ small, the steady-state solution of the extended plant can be made arbitrarily close to the constant performance cost $q_{\mathbf{w}}(u)$, see Fig. 2 . In case of constant or no disturbances $\mathbf{w}$, this difference will be zero (i.e., $\delta_{\mathbf{z} 1}=\delta_{\mathbf{z} 2}=0$ in (16)) for any value of the tuning parameter $\alpha_{\mathbf{z}}$, as the steady-state solution will be independent of time. In case of time-varying disturbances $\mathbf{w}(t)$ (i.e., $\delta_{\mathbf{z} 1}, \delta_{\mathbf{z} 2}>0$ ) the tuning parameter $\alpha_{\mathbf{z}}$ should be tuned small in order to ensure a sufficiently close approximation of the constant performance cost $\mathbf{q}_{\mathbf{w}}(u)$.

Hence, by Assumption 11, under steady-state conditions of the system $\Sigma_{p}$, the cost function $Z$, the filter $\Sigma_{f}$, the limit $\alpha_{\mathbf{z}} \rightarrow 0$, and for constant inputs $\mathbf{u}$, we have that the parameter-to-steady-state performance map of the system can be characterized by

$F_{\mathbf{w}}(\mathbf{u}):=k\left(\mathbf{q}_{\mathbf{w}}(\mathbf{u})\right), \quad \forall \mathbf{u} \in \mathbb{R}^{n_{\mathbf{u}}}$.

We refer to the map $F_{\mathbf{w}}$ as the objective function. To optimize the steady-state system output $\overline{\mathbf{e}}_{\mathbf{w}}$, we aim to find the system parameter values for which the objective function in $(20)$ is minimal. We further assume that, given a disturbance $\mathbf{w}(t) \in \mathcal{W}$, the dynamic cost function is designed such that there exists a unique minimum of the objective function $F_{\mathbf{w}}$.

Assumption 13. Given a disturbance $\mathbf{w}(t) \in \mathcal{W}$, the objective function $F_{\mathbf{w}}: \mathbb{R}^{n_{\mathbf{u}}} \rightarrow \mathbb{R}$ in (20) is twice continuously differentiable and exhibits a unique minimum in $\mathbb{R}^{n_{\mathbf{u}}}$. Let the corresponding optimal input $\mathbf{u}_{\mathbf{w}}^{*}$ be defined as $\mathbf{u}_{\mathbf{w}}^{*}=\arg \min _{\mathbf{u} \in \mathbb{R}^{n_{\mathbf{u}}}} F_{\mathbf{w}}(\mathbf{u})$. There exist constants $L_{F 1}, L_{F 2} \in \mathbb{R}_{>0}$ such that

$\frac{d F_{\mathbf{w}}}{d \mathbf{u}}(\mathbf{u})\left(\mathbf{u}-\mathbf{u}_{\mathbf{w}}^{*}\right) \geq L_{F 1}\left\|\mathbf{u}-\mathbf{u}_{\mathbf{w}}^{*}\right\|^{2}$,

$\left\|\frac{d^{2} F_{\mathbf{w}}}{d \mathbf{u} d \mathbf{u}^{\top}}(\mathbf{u})\right\| \leq L_{F 2}$

for all $\mathbf{u} \in \mathbb{R}^{n_{\mathbf{u}}}$.

Information of the objective function can only be obtained through measured outputs $l$ of the extended plant in (13). The measured output differs from the objective function $F_{\mathbf{w}}$ in (20) in two ways: i) due to the dynamics of the system in (1) and the filter in (9) not being in steady-state, i.e., $\overline{\mathbf{x}}_{\mathbf{w}}(t, \mathbf{u})$ and $\overline{\mathbf{z}}_{\mathbf{w}}\left(t, \mathbf{u}, \alpha_{\mathbf{z}}\right)$, respectively, and ii) due to the presence of time-varying disturbances $\mathbf{w}(t)$ and the design parameter $\alpha_{\mathbf{z}}$ which, in the presence of time-varying disturbances $\mathbf{w}(t)$, is typically designed to be small, but still non-zero and positive. This causes $\overline{\mathbf{z}}_{\mathbf{w}}\left(t, \mathbf{u}, \alpha_{\mathbf{z}}\right)$ to differ from $\mathbf{q}_{\mathbf{w}}(\mathbf{u})$ (Assumption 11). Nevertheless, we aim to steer the inputs $\mathbf{u}$ to their performance optimizing values $\mathbf{u}_{\mathbf{w}}^{*}$ by using the measured extended plant output $l(t)$ as feedback to an extremum-seeking controller that is introduced in the next section.

\section{Extremum-seeking controller}

The extremum-seeking controller is based on the one in Haring (2016, Chapter 2). In contrast with classical ESC approaches as in Krstić and Wang (2000) and Tan et al. (2006), where an estimate of the gradient of the objective function is obtained solely by correlating the measured system performance with a usersupplied dither signal, this extremum-seeking controller exploits both the user-supplied dither signal and the nominal system parameter. This leads to a more accurate gradient estimate, and may lead to a faster convergence (Gelbert, Moeck, Paschereit, \& King, 2012; Guay \& Dochain, 2015; Haring \& Johansen, 2018).

In Section 3.1, a dither signal design is presented, in Section 3.2, a model of the input-to-output behavior of the extended plant is presented to be used as a basis for gradient estimation, and in Section 3.3, a least-squares observer to estimate the state of that model (and therewith the gradient) and a normalized optimizer to steer the system parameters $\mathbf{u}$ to the minimizer $\mathbf{u}_{\mathbf{w}}^{*}$ are presented.

\subsection{Dither signal}

In order to estimate the gradient of the objective function and use this estimated gradient to drive $\mathbf{u}$ towards $\mathbf{u}_{\mathbf{w}}^{*}$ by an optimizer, we supply the following dither signal:

$\mathbf{u}(t)=\hat{\mathbf{u}}(t)+\alpha_{\omega} \omega(t)$,

where $\alpha_{\omega} \omega$ is a vector of perturbation signals with amplitude $\alpha_{\omega} \in \mathbb{R}_{>0}$, and $\hat{\mathbf{u}}$ is referred to as the nominal system parameter to be generated by the extremum-seeking controller. The vector $\boldsymbol{\omega}$ is defined by $\boldsymbol{\omega}(t)=\left[\omega_{1}(t), \omega_{2}(t), \ldots, \omega_{n_{\mathbf{u}}}(t)\right]^{\top}$, with

$\omega_{i}(t)= \begin{cases}\sin \left(\frac{i+1}{2} \eta_{\omega} t\right), & \text { if } i \text { is odd, } \\ \cos \left(\frac{i}{2} \eta_{\omega} t\right), & \text { if } i \text { is even, }\end{cases}$

for $i=\left\{1,2, \ldots, n_{\mathbf{u}}\right\}$, where $\eta_{\omega} \in \mathbb{R}_{>0}$ is a tuning parameter.

\subsection{Model of input-output behavior of the extended plant}

To obtain an estimate of the gradient of the objective function, we model the input-to-output behavior of the extended plant in (13), that is, from the nominal system parameter input $\hat{\mathbf{u}}$ to the 
measured output of the extended plant $l$, in a general form. Let the state vector of the model be given by

$\mathbf{m}(t)=\left[\begin{array}{ll}F_{\mathbf{w}}(\hat{\mathbf{u}}(t)) & \alpha_{\omega} \frac{d F_{\mathbf{w}}}{d \mathbf{u}}(\hat{\mathbf{u}}(t))\end{array}\right]^{\top}$.

The measured output of the extended plant $l$ in (13) can be written as

$l(t)=k(\mathbf{z}(t))-k\left(\overline{\mathbf{z}}_{\mathbf{w}}\left(t, \mathbf{u}(t), \alpha_{\mathbf{z}}\right)\right)+F_{\mathbf{w}}(\mathbf{u}(t))+d(t)$,

with the signal $d(t)$ defined as

$d(t):=k\left(\overline{\mathbf{z}}_{\mathbf{w}}\left(t, \mathbf{u}(t), \alpha_{\mathbf{z}}\right)\right)-k\left(\mathbf{q}_{\mathbf{w}}(\mathbf{u}(t))\right)$.

Using Taylor's Theorem and (23), the objective function $F_{\mathbf{w}}$ can be written as

$$
\begin{aligned}
F_{\mathbf{w}}(\mathbf{u}(t))= & F_{\mathbf{w}}(\hat{\mathbf{u}}(t))+\alpha \frac{d F_{\mathbf{w}}}{d \mathbf{u}}(\hat{\mathbf{u}}(t)) \boldsymbol{\omega}(t) \\
& +\frac{1}{2} \alpha_{\omega^{2}}^{2} \boldsymbol{\omega}^{\top}(t) \mathbf{H}(t, \hat{\mathbf{u}}(t)) \boldsymbol{\omega}(t),
\end{aligned}
$$

where $\mathbf{H}(t, \hat{\mathbf{u}}(t))$ reads

$\mathbf{H}(t, \hat{\mathbf{u}}(t))=2 \int_{0}^{1}(1-\sigma) \frac{d^{2} F_{\mathbf{w}}}{d \mathbf{u} d \mathbf{u}^{\top}}\left(\hat{\mathbf{u}}(t)+\sigma \alpha_{\omega} \boldsymbol{\omega}(t)\right) d \sigma$.

The dynamics of the state vector in (25) is governed by

$$
\begin{aligned}
\dot{\mathbf{m}}(t) & =\mathbf{A}(t) \mathbf{m}(t)+\alpha_{\omega}^{2} \mathbf{B s}(t) \\
l(t) & =\mathbf{C}(t) \mathbf{m}(t)+\alpha_{\omega}^{2} v(t)+r(t)+d(t),
\end{aligned}
$$

with the matrices $\mathbf{A}, \mathbf{B}$ and $\mathbf{C}$ defined as

$$
\mathbf{A}(t)=\frac{1}{\alpha_{\omega}}\left[\begin{array}{cc}
0 & \dot{\hat{\mathbf{u}}}^{\top}(t) \\
\mathbf{0}^{n_{\mathbf{u}} \times 1} & \mathbf{0}^{n_{\mathbf{u}} \times n_{\mathbf{u}}}
\end{array}\right], \mathbf{B}=\left[\begin{array}{c}
\mathbf{0}^{1 \times n_{\mathbf{u}}} \\
\mathbf{I}^{n_{\mathbf{u}} \times n_{\mathbf{u}}}
\end{array}\right], \mathbf{C}=\left[\begin{array}{c}
1 \\
\omega(t)
\end{array}\right]^{\top},
$$

and the signals $\mathbf{S}, v$, and $r$ defined as

$$
\begin{aligned}
\mathbf{s}(t) & :=\frac{d^{2} F_{\mathbf{w}}}{d \mathbf{u} d \mathbf{u}^{\top}}(\hat{\mathbf{u}}(t)) \frac{\dot{\hat{\mathbf{u}}}(t)}{\alpha_{\boldsymbol{\omega}}}, v(t):=\frac{1}{2} \boldsymbol{\omega}^{\top}(t) \mathbf{H}(t, \hat{\mathbf{u}}(t)) \boldsymbol{\omega}(t), \\
r(t) & :=k(\mathbf{z}(t))-k\left(\overline{\mathbf{z}}_{\mathbf{w}}\left(t, \mathbf{u}(t), \alpha_{\mathbf{z}}\right)\right) .
\end{aligned}
$$

The signals $\mathbf{s}, v, r$, and $d$ can be interpreted as unknown disturbances to the model in (30). The influences of $\mathbf{s}, v, r$, and $d$ on the state and output of the model in (30) are small if (i) $\hat{\mathbf{u}}$ is slowly time varying, if (ii) $\alpha_{\omega}$ is small, if (iii) the states $\mathbf{x}$ of the system in (1) and the states $\mathbf{z}$ of the filter in (9) are close to their steady-state values, respectively $\overline{\mathbf{x}}_{\mathbf{w}}$ and $\overline{\mathbf{z}}_{\mathbf{w}}$, and if (iv) $\alpha_{\mathbf{z}}$ is small.

The state $\mathbf{m}$ in (25) contains the gradient of the objective function, scaled by the perturbation amplitude $\alpha_{\omega}$. Hence, an estimate of the gradient of the objective function can be obtained from an estimate of the state $\mathbf{m}$.

\subsection{Controller design}

The extremum-seeking controller is composed of a dither signal as in (23), a least-squares observer to estimate the state $\mathbf{m}$ of the model in (30), and an optimizer that uses the estimate of the state $\mathbf{m}$ of the observer, denoted by $\hat{\mathbf{m}}$, to steer the nominal system inputs $\hat{\mathbf{u}}$ to their performance optimal values $\mathbf{u}_{\mathbf{w}}^{*}$.

The least-squares observer, denoted by $\Sigma_{0}$, is given by

$$
\Sigma_{o}:\left\{\begin{aligned}
\dot{\hat{\mathbf{m}}}= & \left(\mathbf{A}-\eta_{\mathbf{m}} \sigma_{r} \mathbf{Q} \mathbf{D}^{\top} \mathbf{D}\right) \hat{\mathbf{m}}+\alpha_{\omega}^{2} \mathbf{B} \hat{\mathbf{s}} \\
& +\eta_{\mathbf{m}} \mathbf{Q} \mathbf{C}^{\top}\left(l-\mathbf{C} \hat{\mathbf{m}}-\alpha_{\omega}^{2} \hat{v}\right) \\
\dot{\mathbf{Q}}= & \eta_{\mathbf{m}} \mathbf{Q}+\mathbf{A Q}+\mathbf{Q} \mathbf{A}^{\top} \\
& -\eta_{\mathbf{m}} \mathbf{Q}\left(\mathbf{C}^{\top} \mathbf{C}+\sigma_{r} \mathbf{D}^{\top} \mathbf{D}\right) \mathbf{Q},
\end{aligned}\right.
$$

with initial conditions $\hat{\mathbf{m}}(0)=\hat{\mathbf{m}}_{0} \in \mathbb{R}^{n_{\mathbf{u}}+1}$ and $\mathbf{Q}(0)=\mathbf{Q}_{0} \in$ $\mathbb{R}^{n_{\mathbf{u}}+1 \times n_{\mathbf{u}}+1}$, where $\mathbf{Q}_{0}$ is a symmetric and positive definite matrix, $\mathbf{D}=\left[\begin{array}{ll}\mathbf{0}^{n_{\mathbf{u} \times 1}} & \mathbf{I}^{n_{\mathbf{u}} \times n_{\mathbf{u}}}\end{array}\right], \eta_{\mathbf{m}} \in \mathbb{R}_{>0}$ and $\sigma_{r} \in \mathbb{R}_{\geq 0}$ are tuning parameters related to the observer, referred to as a forgetting factor and a regularization constant, respectively, and signals $\hat{\mathbf{s}}$ and $\hat{v}$ being approximations of signals $\mathbf{s}$ and $v$ in (32), defined as

$\hat{\mathbf{s}}:=\hat{\mathbf{H}}(t, \hat{\mathbf{u}}(t)) \frac{\dot{\hat{\mathbf{u}}}(t)}{\alpha_{\omega}}, \hat{v}:=\frac{1}{2} \boldsymbol{\omega}^{\top}(t) \hat{\mathbf{H}}(t, \hat{\mathbf{u}}(t)) \boldsymbol{\omega}(t)$,

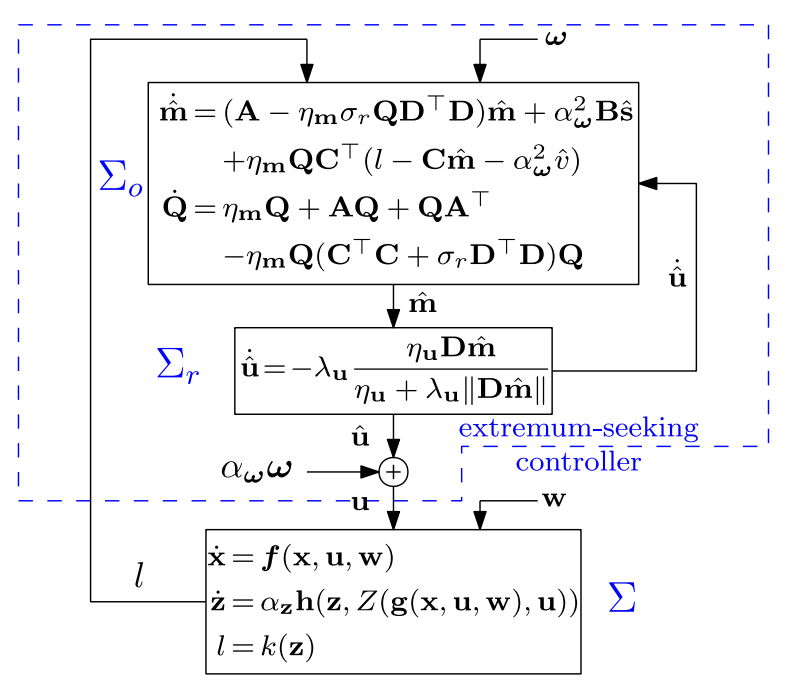

Fig. 3. The closed-loop system composed of the extended plant $\Sigma$, the observer $\Sigma_{0}$, the optimizer $\Sigma_{r}$, and the dither signal $\alpha_{\omega} \omega$.

with a user-defined function $\hat{\mathbf{H}}: \mathbb{R} \times \mathbb{R}^{n_{\mathbf{u}}} \rightarrow \mathbb{R}^{n_{\mathbf{u}} \times n_{\mathbf{u}}}$ satisfying $\|\hat{\mathbf{H}}(t, \hat{\mathbf{u}})\| \leq L_{\mathbf{H}}$, for all $t \in \mathbb{R}$, all $\hat{\mathbf{u}} \in \mathbb{R}^{n_{\mathbf{u}}}$, and with $L_{\mathbf{H}} \in \mathbb{R}_{>0}$.

The optimizer, denoted by $\Sigma_{r}$, is given by

$\Sigma_{r}: \quad \dot{\hat{\mathbf{u}}}(t)=-\lambda_{\mathbf{u}} \frac{\eta_{\mathbf{u}} \mathbf{D} \hat{\mathbf{m}}(t)}{\eta_{\mathbf{u}}+\lambda_{\mathbf{u}}\|\mathbf{D} \hat{\mathbf{m}}(t)\|}$,

with $\lambda_{\mathbf{u}}, \eta_{\mathbf{u}} \in \mathbb{R}_{>0}$ being tuning parameters related to the optimizer. Normalization of the adaptation gain in (35) is done to prevent solutions of the closed-loop ESC scheme from having a finite escape time if the state estimate $\hat{\mathbf{m}}$ is inaccurate (Haring, 2016, Chapter 2). The closed-loop system, composed of the extended plant $\Sigma$ in (13), the observer $\Sigma_{0}$ in (33), and the optimizer $\Sigma_{r}$ in (35), is depicted in Fig. 3.

\section{Stability analysis}

In this section, we will provide a stability result and supporting stability proof for the closed-loop system described in the previous sections. The next result states conditions on tuning parameters under which the ESC scheme guarantees that $\hat{\mathbf{u}}$ converges to an arbitrarily small set around the optimum $\mathbf{u}_{\mathbf{w}}^{*}$.

Theorem 14. Consider a (time-varying) disturbance $\mathbf{w} \in \mathcal{W}$ and Assumptions 1, 5, 11, and 13. Moreover, consider arbitrary initial conditions $\mathbf{x}(0) \in \mathbb{R}^{n_{\mathbf{x}}}, \mathbf{Q}(0) \in \mathbb{R}^{n_{\mathbf{u}}+1 \times n_{\mathbf{u}}+1}$ symmetric and positive-definite, $\mathbf{z}(0) \in \mathbb{R}^{n_{\mathbf{z}}}, \hat{\mathbf{m}}(0) \in \mathbb{R}^{n_{\mathbf{u}}+1}$, and an arbitrary compact set $\mathcal{U}_{0} \subset \mathbb{R}^{n_{\mathbf{u}}}$ of initial conditions for $\hat{\mathbf{u}}(t)$. Then, there exist sufficiently small constants $\epsilon_{0}, \ldots, \epsilon_{6} \in \mathbb{R}_{>0}$ such that, for all tunable parameters $\alpha_{\mathbf{z}}, \alpha_{\omega}, \eta_{\mathbf{u}}, \lambda_{\mathbf{u}}, \eta_{\mathbf{m}}, \eta_{\omega} \in \mathbb{R}_{>0}$ and $\sigma_{r} \in \mathbb{R}_{\geq 0}$ with $\alpha_{\omega} \leq \epsilon_{0}, \alpha_{\mathbf{z}} \leq \alpha_{\omega} \epsilon_{1}, \eta_{\omega} \leq \alpha_{\mathbf{z}} \epsilon_{2}, \eta_{\mathbf{m}} \leq \eta_{\omega} \epsilon_{3}, \alpha_{\omega} \lambda_{\mathbf{u}} \leq$ $\eta_{\mathbf{m}} \epsilon_{4}, \quad \eta_{\mathbf{u}} \leq \alpha_{\omega} \eta_{\mathbf{m}} \epsilon_{5}$, and $\sigma_{r} \leq \epsilon_{6}$, the solutions of the closed-loop system consisting of the extended plant in (13) and the extremumseeking controller (consisting of the dither signal in (23), the observer $\Sigma_{0}$ in (33), and the optimizer $\Sigma_{r}$ in (35)) are bounded for all $t \geq 0$. In addition, there exist constants $c_{1}, \ldots, c_{4} \in \mathbb{R}_{>0}$ such that the solutions $\hat{\mathbf{u}}(t)$ with $\hat{\mathbf{u}}(0) \in \mathcal{U}_{0}$ satisfy

$$
\begin{array}{r}
\limsup _{t \rightarrow \infty}\left\|\hat{\mathbf{u}}(t)-\mathbf{u}_{\mathbf{w}}^{*}\right\| \leq \\
\max \left\{\alpha_{\omega} c_{1}, \frac{\eta_{\omega}}{\alpha_{\mathbf{z}}} c_{2},\right. \\
\left.\frac{\alpha_{\mathbf{z}} \delta_{\mathbf{z} 1}}{\alpha_{\boldsymbol{\omega}}} c_{3}, \alpha_{\mathbf{z}} \alpha_{\boldsymbol{\omega}} \delta_{\mathbf{z} 2} c_{4}\right\},
\end{array}
$$

Remark 15. Tuning guidelines. Under the conditions of Theorem 14 it follows that, if we are dealing with constant 
(or no) disturbances $\mathbf{w}$, i.e., $\delta_{\mathbf{z} 1}, \delta_{\mathbf{z} 2}=0$ (Assumption 11), the optimizer state $\hat{\mathbf{u}}$ converges to an arbitrarily small region of the performance-optimal value $\mathbf{u}_{\mathbf{w}}^{*}$ if the dither parameters $\alpha_{\omega}$ and $\eta_{\omega}$ are chosen sufficiently small for an arbitrary bounded $\alpha_{\mathbf{z}}$. Choosing $\alpha_{\mathbf{z}}$ large in general allows faster convergence towards the performance-optimal value $\mathbf{u}_{\mathbf{w}}^{*}$. In the case of time-varying disturbances $\mathbf{w}(t)$, i.e., $\delta_{\mathbf{z} 1}, \delta_{\mathbf{z} 2}>0$, see (36), we subsequently tune $\alpha_{\omega}$ small to make the first term on the right-hand side of (36) arbitrarily small, tune $\alpha_{\mathbf{z}}$ small to make the third and fourth term on the right-hand side of (36) arbitrarily small, and finally tune $\eta_{\omega}$ small to make the second term on the right-hand side of (36) arbitrarily small.

Proof. To prove Theorem 14, we introduce the following coordinate transformation:

$$
\begin{aligned}
\tilde{\mathbf{x}}(t) & =\mathbf{x}(t)-\overline{\mathbf{x}}_{\mathbf{w}}(t, \mathbf{u}(t)), \\
\tilde{\mathbf{z}}(t) & =\mathbf{z}(t)-\overline{\mathbf{z}}_{\mathbf{w}}\left(t, \mathbf{u}(t), \alpha_{\mathbf{z}}\right), \\
\tilde{\mathbf{u}}(t) & =\hat{\mathbf{u}}(t)-\mathbf{u}_{\mathbf{w}}^{*}, \\
\tilde{\mathbf{m}}(t) & =\hat{\mathbf{m}}(t)-\mathbf{m}(t), \\
\tilde{\mathbf{Q}}(t) & =\mathbf{Q}^{-1}(t)-\Xi^{-1}-\eta_{\mathbf{m}} \mathbf{n}(t),
\end{aligned}
$$

with

$$
\begin{aligned}
& \mathbf{n}(t)=\int_{0}^{t}\left[\begin{array}{ll}
0 & \boldsymbol{\omega}^{\top}(\tau) \\
\omega(\tau) & \boldsymbol{\omega}(\tau) \boldsymbol{\omega}^{\top}(\tau)-\frac{1}{2} \mathbf{I}
\end{array}\right] d \tau, \\
& \Xi=\left[\begin{array}{cc}
1 & \mathbf{0}^{1 \times n_{\mathbf{u}}} \\
\mathbf{0}^{n_{\mathbf{u}} \times 1} & \frac{2}{1+2 \sigma_{r}} \mathbf{I}^{n_{\mathbf{u}} \times n_{\mathbf{u}}}
\end{array}\right] .
\end{aligned}
$$

Let us define the following vector fields

$$
\begin{aligned}
& \tilde{\mathbf{f}}_{\mathbf{w}}(t, \tilde{\mathbf{x}}, \mathbf{u}):=\mathbf{f}\left(\tilde{\mathbf{x}}+\overline{\mathbf{x}}_{\mathbf{w}}(t, \mathbf{u}), \mathbf{u}, \mathbf{w}(t)\right) \\
& -\mathbf{f}\left(\overline{\mathbf{x}}_{\mathbf{w}}(t, \mathbf{u}), \mathbf{u}, \mathbf{w}(t)\right), \\
& \tilde{\mathbf{h}}_{\mathbf{w}}\left(t, \tilde{\mathbf{z}}, \mathbf{u}, \alpha_{\mathbf{z}}\right):=\mathbf{h}\left(\tilde{\mathbf{z}}+\overline{\mathbf{z}}_{\mathbf{w}}\left(t, \mathbf{u}, \alpha_{\mathbf{z}}\right), \bar{y}_{\mathbf{w}}(t, \mathbf{u})\right) \\
& -\mathbf{h}\left(\overline{\mathbf{z}}_{\mathbf{w}}\left(t, \mathbf{u}, \alpha_{\mathbf{z}}\right), \bar{y}_{\mathbf{w}}(t, \mathbf{u})\right) \text {. }
\end{aligned}
$$

The analysis of the stability properties of the closed-loop system can be divided into two temporal stages, defined by some finite time instance $t_{1}$ : (i) for $0 \leq t<t_{1}$, the solutions $\tilde{\mathbf{x}}$ and $\tilde{\mathbf{Q}}$ converge to a neighborhood of the origin and remain there, while the solutions $\tilde{\mathbf{z}}, \tilde{\mathbf{m}}$, and $\tilde{\mathbf{u}}$ may drift, but remain bounded, and (ii) for $t \geq t_{1}$, the solutions $\tilde{\mathbf{z}}, \tilde{\mathbf{m}}$, and $\tilde{\mathbf{u}}$ also converge to a neighborhood of the origin.

We first derive bounds on each of the variables in (37) corresponding to these two temporal stages of convergence. To derive a bound on the solutions $\tilde{\mathbf{x}}(t)$ for time-varying inputs $\mathbf{u}(t)$, a preliminary result is presented in Lemma 16 on the existence of a Lyapunov function for the $\tilde{\mathbf{x}}$-dynamics for constant inputs $\mathbf{u}$.

Lemma 16. Under Assumptions 1 and 5, there exist a function $V_{\mathbf{x}}: \mathbb{R} \times \mathbb{R}^{n_{\mathbf{x}}} \times \mathbb{R}^{n_{\mathbf{u}}} \rightarrow \mathbb{R}$, and constants $\gamma_{\mathbf{x} 1}, \ldots, \gamma_{\mathbf{x} 5} \in \mathbb{R}_{>0}$, such that the inequalities

$\gamma_{\mathbf{x} 1}\|\tilde{\mathbf{x}}\|^{2} \leq V_{\mathbf{x}}(t, \tilde{\mathbf{x}}, \mathbf{u}) \leq \gamma_{\mathbf{x} 2}\|\tilde{\mathbf{x}}\|^{2}$,

$\frac{\partial V_{\mathbf{x}}}{\partial t}(t, \tilde{\mathbf{x}}, \mathbf{u})+\frac{\partial V_{\mathbf{x}}}{\partial \tilde{\mathbf{x}}}(t, \tilde{\mathbf{x}}, \mathbf{u}) \tilde{\mathbf{f}}_{\mathbf{w}}(t, \tilde{\mathbf{x}}, \mathbf{u}) \leq-\gamma_{\mathbf{x} 3}\|\tilde{\mathbf{x}}\|^{2}$,

$\left\|\frac{\partial V_{\mathbf{x}}}{\partial \tilde{\mathbf{x}}}(t, \tilde{\mathbf{x}}, \mathbf{u})\right\| \leq \gamma_{\mathbf{x} 4}\|\tilde{\mathbf{x}}\|,\left\|\frac{\partial V_{\mathbf{x}}}{\partial \mathbf{u}}(t, \tilde{\mathbf{x}}, \mathbf{u})\right\| \leq \gamma_{\mathbf{x} 5}\|\tilde{\mathbf{x}}\|$,

are satisfied for all $t \in \mathbb{R}$, all $\tilde{\mathbf{x}} \in \mathbb{R}^{n_{\mathbf{x}}}$, and all constant $\mathbf{u} \in \mathbb{R}^{n_{\mathbf{u}}}$.

Proof. The proof of Lemma 16 follows a similar line of reasoning as Theorem 4.14 and Lemma 9.8 in Khalil (2002). Different from the results in Khalil (2002) is the second inequality in (43); this stems from the assumption of uniform boundedness of $\left[\partial \tilde{\mathbf{f}}_{\mathbf{w}} / \partial \mathbf{u}\right]$, i.e., $\left\|\partial \tilde{\mathbf{f}}_{\mathbf{w}} / \partial \mathbf{u}\right\| \leq 2 L_{\mathbf{f u}}$, which follows from Assumption 1 and the coordinate transformation in (37).
From the system $\Sigma_{p}$ in (1), the coordinate transformation in (37), and the vector field defined by (40), it follows that the state equation for $\tilde{\mathbf{x}}$ for time-varying inputs $\mathbf{u}(t)$, generated by (35) and (23), is given by

$\dot{\tilde{\mathbf{x}}}(t)=\tilde{\mathbf{f}}_{\mathbf{w}}(t, \tilde{\mathbf{x}}(t), \mathbf{u}(t))-\frac{\partial \overline{\mathbf{x}}_{\mathbf{w}}}{\partial \mathbf{u}}(t, \mathbf{u}(t)) \dot{\mathbf{u}}(t)$.

A bound on the solutions of the $\tilde{\mathbf{x}}$-dynamics for time-varying inputs $\mathbf{u}(t)$ in (44) is presented in Lemma 17.

Lemma 17. Under Assumptions 1 and 5, for any $\epsilon_{3}, \epsilon_{5} \in \mathbb{R}_{>0}$, and all tunable parameters $\alpha_{\omega}, \eta_{\omega} \in \mathbb{R}_{>0}$, there exist constants $c_{\mathbf{x} 1}, c_{\mathbf{x} 2}\left(\epsilon_{3}, \epsilon_{5}\right), \beta_{\mathbf{x}} \in \mathbb{R}_{>0}$ such that, for all $\eta_{\mathbf{m}} \leq \eta_{\omega} \epsilon_{3}$ and all $\eta_{\mathbf{u}} \leq \alpha_{\omega} \eta_{\mathbf{m}} \epsilon_{5}$, the solutions $\tilde{\mathbf{x}}$ satisfy

$\|\tilde{\mathbf{x}}(t)\| \leq \max \left\{c_{\mathbf{x} 1}\|\tilde{\mathbf{x}}(0)\| e^{-\beta_{\mathbf{x}} t}, \alpha_{\omega} \eta_{\omega} c_{\mathbf{x} 2}\right\}$,

for all $t \geq 0$, all $\tilde{\mathbf{x}}(0) \in \mathbb{R}^{n_{\mathbf{x}}}$, and all time-varying $\mathbf{u}(t) \in \mathbb{R}^{n_{\mathbf{u}}}$.

Proof. See Appendix A.

From the observer in (33), the coordinate transformation in (37), and the model of the input-output behavior in (30), we obtain that the state equation for $\tilde{\mathbf{Q}}$ is given by

$$
\begin{aligned}
\dot{\tilde{\mathbf{Q}}}(t)= & -\eta_{\mathbf{m}} \tilde{\mathbf{Q}}(t)-\tilde{\mathbf{Q}}(t) \mathbf{A}(t)-\mathbf{A}^{\top}(t) \tilde{\mathbf{Q}}(t)-\eta_{\mathbf{m}}^{2} \mathbf{n}(t) \\
& -\left(\Xi^{-1}+\eta_{\mathbf{m}} \mathbf{n}(t)\right) \mathbf{A}(t)-\mathbf{A}^{\top}(t)\left(\Xi^{-1}+\eta_{\mathbf{m}} \mathbf{n}(t)\right) .
\end{aligned}
$$

A bound on the solutions $\tilde{\mathbf{Q}}(t)$ is presented in Lemma 18 .

Lemma 18. For sufficiently small $\epsilon_{3}, \epsilon_{5}, \epsilon_{6} \in \mathbb{R}_{>0}$ and all tunable parameters $\alpha_{\omega}, \eta_{\omega} \in \mathbb{R}_{>0}$, there exist constants $c_{\mathbf{Q}}, \beta_{\mathbf{Q}} \in \mathbb{R}_{>0}$ such that, for all $\eta_{\mathbf{m}} \leq \eta_{\omega} \epsilon_{3}$, all $\eta_{\mathbf{u}} \leq \alpha_{\omega} \eta_{\mathbf{m}} \epsilon_{5}$, and all $\sigma_{r} \leq \epsilon_{6}$, the solutions $\tilde{\mathbf{Q}}$ satisfy

$\|\tilde{\mathbf{Q}}(t)\| \leq \max \left\{c_{\mathbf{Q}}\|\tilde{\mathbf{Q}}(0)\| e^{-\eta_{\mathbf{m}} \beta_{\mathbf{Q}} t}, \frac{1}{8}\right\}$,

for all $t \geq 0$, all $\tilde{\mathbf{Q}}(0) \in \mathbb{R}^{n_{\mathbf{u}}+1 \times n_{\mathbf{u}}+1}$ for which $\mathbf{Q}(0)$ is symmetric and positive definite, and all time-varying $\mathbf{u}(t) \in \mathbb{R}^{n_{\mathbf{u}}}$.

Proof. See the proof of Lemma 2.11 in Haring (2016).

From Lemmas 17 and 18, we conclude that there exists a finite time $t_{1} \geq 0$ such that $\|\tilde{\mathbf{x}}(t)\| \leq \alpha_{\omega} \eta_{\omega} c_{\mathbf{x} 2}$ and $\|\tilde{\mathbf{Q}}(t)\| \leq \frac{1}{8}$ for all $t \geq t_{1}$. These bounds on $\tilde{\mathbf{x}}(t)$ and $\tilde{\mathbf{Q}}(t)$ are utilized to obtain bounds on the solutions $\tilde{\mathbf{u}}(t), \tilde{\mathbf{z}}(t)$, and $\tilde{\mathbf{m}}(t)$ in Lemmas 19, 21 , and 22 , respectively.

Firstly, consider the $\tilde{\mathbf{u}}$-dynamics. From the optimizer in (35) and the coordinate transformation in (37), it follows that the state equation for $\tilde{\mathbf{u}}$ for time-varying inputs $\mathbf{u}(t)$ is given by

$\dot{\tilde{\mathbf{u}}}(t)=-\lambda_{\mathbf{u}} \frac{\eta_{\mathbf{u}} \mathbf{D}(\tilde{\mathbf{m}}(t)+\mathbf{m}(t))}{\eta_{\mathbf{u}}+\lambda_{\mathbf{u}}\|\mathbf{D}(\tilde{\mathbf{m}}(t)+\mathbf{m}(t))\|}$.

A bound on the solutions $\tilde{\mathbf{u}}(t)$ for time-varying inputs $\mathbf{u}(t)$ in (48) is presented in Lemma 19.

Lemma 19. Under Assumption 13 , for any finite time $t_{1} \geq 0$, and all tunable parameters $\eta_{\mathbf{u}}, \lambda_{\mathbf{u}}, \alpha_{\omega} \in \mathbb{R}_{>0}$, the solutions $\tilde{\mathbf{u}}$ are bounded for all $0 \leq t \leq t_{1}$, and all $\tilde{\mathbf{u}}(0) \in \mathbb{R}^{n_{\mathbf{u}}}$. In addition, there exists $a$ constant $c_{\mathbf{u} 1} \in \mathbb{R}_{>0}$ such that the solutions $\tilde{\mathbf{u}}$ satisfy

$$
\begin{aligned}
& \sup _{t \geq t_{1}}\|\tilde{\mathbf{u}}(t)\| \leq \max \left\{\left\|\tilde{\mathbf{u}}\left(t_{1}\right)\right\|, \frac{1}{\alpha_{\omega}} c_{\mathbf{u} 1} \sup _{t \geq t_{1}}\|\tilde{\mathbf{m}}(t)\|\right\}, \\
& \limsup _{t \rightarrow \infty}\|\tilde{\mathbf{u}}(t)\| \leq \frac{1}{\alpha_{\omega}} c_{\mathbf{u} 1} \limsup _{t \rightarrow \infty}\|\tilde{\mathbf{m}}(t)\| .
\end{aligned}
$$

Proof. See the proof of Lemma 2.12 in Haring (2016). 
Secondly, consider the $\tilde{\mathbf{z}}$-dynamics. To derive a bound on the solutions $\tilde{\mathbf{z}}(t)$ for time-varying inputs $\mathbf{u}(t)$, a preliminary result is presented in Lemma 20 on the existence of a Lyapunov function for the $\tilde{\mathbf{z}}$-dynamics for constant inputs $\mathbf{u}$.

Lemma 20. Under Assumptions 1, 5, 11, given (10), (12), and Property 8, there exist a function $V_{\mathbf{z}}: \mathbb{R} \times \mathbb{R}^{n_{\mathbf{z}}} \times \mathbb{R}^{n_{\mathbf{u}}} \times \mathbb{R} \rightarrow \mathbb{R}$, and constants $\gamma_{\mathbf{z} 1}, \ldots, \gamma_{\mathbf{z} 6} \in \mathbb{R}_{>0}$, such that the inequalities

$$
\begin{aligned}
& \gamma_{\mathbf{z} 1}\|\tilde{\mathbf{z}}\|^{2} \leq V_{\mathbf{z}}\left(t, \tilde{\mathbf{z}}, \mathbf{u}, \alpha_{\mathbf{z}}\right) \leq \gamma_{\mathbf{z} 2}\|\tilde{\mathbf{z}}\|^{2}, \\
& \frac{\partial V_{\mathbf{z}}}{\partial t}\left(t, \tilde{\mathbf{z}}, \mathbf{u}, \alpha_{\mathbf{z}}\right)+\alpha_{\mathbf{z}} \frac{\partial V_{\mathbf{z}}}{\partial \tilde{\mathbf{z}}}\left(t, \tilde{\mathbf{z}}, \mathbf{u}, \alpha_{\mathbf{z}}\right) \tilde{\mathbf{h}}_{\mathbf{w}}\left(t, \tilde{\mathbf{z}}, \mathbf{u}, \alpha_{\mathbf{z}}\right) \\
& \left\|\frac{\partial V_{\mathbf{z}}}{\partial \tilde{\mathbf{z}}}\left(t, \tilde{\mathbf{z}}, \mathbf{u}, \alpha_{\mathbf{z}}\right)\right\| \leq \gamma_{\mathbf{z} 3}\|\tilde{\mathbf{z}}\|^{2}, \\
& \left\|\frac{\partial V_{\mathbf{z}}\|\tilde{\mathbf{z}}\|,}{\partial \mathbf{u}}\left(t, \tilde{\mathbf{z}}, \mathbf{u}, \alpha_{\mathbf{z}}\right)\right\| \leq \gamma_{\mathbf{z} 5}\|\tilde{\mathbf{z}}\|+\gamma_{\mathbf{z} 6}\|\tilde{\mathbf{z}}\|\left\|\mathbf{u}-\mathbf{u}_{\mathbf{w}}^{*}\right\|,
\end{aligned}
$$

are satisfied for all $t \in \mathbb{R}$, all $\tilde{\mathbf{z}} \in \mathbb{R}^{n_{\mathbf{z}}}$, all $\alpha_{\mathbf{z}} \in \mathbb{R}_{>0}$, and all constant $\mathbf{u}, \mathbf{u}_{\mathbf{w}}^{*} \in \mathbb{R}^{n_{\mathbf{u}}}$.

Proof. The proof of Lemma 20 follows a similar line of reasoning as Theorem 4.14 and Lemma 9.8 in Khalil (2002). Different from the results in Khalil (2002) is the inequality in (54); this stems from the bound on $\left[\partial \tilde{\mathbf{h}}_{\mathbf{w}} / \partial \mathbf{u}\right]$, i.e., $\left\|\partial \tilde{\mathbf{h}}_{\mathbf{w}} / \partial \mathbf{u}\right\| \leq L_{\mathbf{h z}}\left(\epsilon_{\mathbf{z}} L_{\mathbf{z} 1}+\right.$ $\left.\left(\epsilon_{\mathbf{z}} L_{\mathbf{z} 2}+L_{\mathbf{q}}\right)\left\|\mathbf{u}-\mathbf{u}_{\mathbf{w}}^{*}\right\|\right)$, resulting from (12), Assumption 11, and the coordinate transformation in (37).

From the filter $\Sigma_{f}$ in (9), the coordinate transformation in (37), and the vector field defined by (40), it follows that the state equation for $\tilde{\mathbf{z}}$ for time-varying inputs $\mathbf{u}(t)$, generated by (23) and (35), is given by

$$
\begin{aligned}
\dot{\tilde{\mathbf{z}}}(t)= & \alpha_{\mathbf{z}} \tilde{\mathbf{h}}_{\mathbf{w}}\left(t, \tilde{\mathbf{z}}(t), \mathbf{u}(t), \alpha_{\mathbf{z}}\right)-\frac{\partial \overline{\mathbf{z}}_{\mathbf{w}}}{\partial \mathbf{u}}\left(t, \mathbf{u}(t), \alpha_{\mathbf{z}}\right) \dot{\mathbf{u}}(t) \\
& +\alpha_{\mathbf{z}}\left(\mathbf{h}(\mathbf{z}(t), y(t))-\mathbf{h}\left(\mathbf{z}(t), \bar{y}_{\mathbf{w}}(t, \mathbf{u}(t))\right)\right) .
\end{aligned}
$$

A bound on the solutions of the $\tilde{\mathbf{z}}$-dynamics for time-varying inputs $\mathbf{u}(t)$ in (55) is presented in Lemma 21.

Lemma 21. Under Assumptions 1, 5, 11, given (10), (12), and Property 8 , for any $\epsilon_{0}, \ldots, \epsilon_{3}, \epsilon_{5}, \epsilon_{\mathbf{z}} \in \mathbb{R}_{>0}$, any finite time $t_{1} \geq 0$, and all $\alpha_{\omega} \leq \epsilon_{0}, \alpha_{\mathbf{z}} \leq \alpha_{\omega} \epsilon_{1} \leq \epsilon_{\mathbf{z}}, \eta_{\omega} \leq \alpha_{\mathbf{z}} \epsilon_{2}, \eta_{\mathbf{m}} \leq \eta_{\omega} \epsilon_{3}, \eta_{\mathbf{u}} \leq$ $\alpha_{\omega} \eta_{\mathbf{m}} \epsilon_{5}$, the solutions $\tilde{\mathbf{z}}$ are bounded for all $0 \leq t \leq t_{1}$, all $\tilde{\mathbf{z}}(0) \in$ $\mathbb{R}^{n_{\mathbf{z}}}$, all $\tilde{\mathbf{x}}(0) \in \mathbb{R}^{n_{\mathbf{x}}}$, and all $\tilde{\mathbf{u}}(0) \in \mathbb{R}^{n_{\mathbf{u}}}$. In addition, there exist constants $\quad c_{\mathbf{z} 1}, c_{\mathbf{z} 2}\left(\epsilon_{0}, \epsilon_{1}, \epsilon_{3}, \epsilon_{5}\right), c_{\mathbf{z} 3}\left(\epsilon_{0}, \ldots, \epsilon_{3}, \epsilon_{5}, \epsilon_{\mathbf{z}}\right)$, $c_{\mathbf{z} 4}\left(\epsilon_{0}, \epsilon_{1}, \epsilon_{3}, \epsilon_{5}, \epsilon_{\mathbf{z}}\right) \in \mathbb{R}_{>0}$ such that the solutions $\tilde{\mathbf{z}}$ satisfy

$$
\begin{gathered}
\sup _{t \geq t_{1}}\|\tilde{\mathbf{z}}(t)\| \leq \sup _{t \geq t_{1}} \max \left\{c_{\mathbf{z} 1}\left\|\tilde{\mathbf{z}}\left(t_{1}\right)\right\|, \frac{\alpha_{\omega} \eta_{\omega}}{\alpha_{\mathbf{z}}} c_{\mathbf{z} 2},\right. \\
\left.\alpha_{\omega}^{2} c_{\mathbf{z} 3}, \frac{\alpha_{\omega} \eta_{\omega}}{\alpha_{\mathbf{z}}} c_{\mathbf{z} 4}\|\tilde{\mathbf{u}}(t)\|\right\}, \\
\limsup _{t \rightarrow \infty}\|\tilde{\mathbf{z}}(t)\| \leq \limsup _{t \rightarrow \infty} \max \left\{\frac{\alpha_{\omega} \eta_{\omega}}{\alpha_{\mathbf{z}}} c_{\mathbf{z} 2}, \alpha_{\omega}^{2} c_{\mathbf{z} 3},\right. \\
\left.\frac{\alpha_{\omega} \eta_{\omega}}{\alpha_{\mathbf{z}}} c_{\mathbf{z} 4}\|\tilde{\mathbf{u}}(t)\|\right\},
\end{gathered}
$$

for all $t \geq t_{1}$, and all time-varying $\tilde{\mathbf{u}}(t) \in \mathbb{R}^{n_{\mathbf{u}}}$.

\section{Proof. See Appendix B.}

Thirdly, consider the $\tilde{\mathbf{m}}$-dynamics. From the observer in (33), the coordinate transformation in (37), the model of the inputoutput behavior in (30), and the state definition in (25), we obtain that the state equation for $\tilde{\mathbf{m}}$ is given by

$$
\begin{aligned}
\dot{\tilde{\mathbf{m}}}(t)= & \left(\mathbf{A}(t)-\eta_{\mathbf{m}} \mathbf{Q}(t)\left(\mathbf{C}^{\top}(t) \mathbf{C}(t)+\sigma_{r} \mathbf{D}^{\top} \mathbf{D}\right)\right) \tilde{\mathbf{m}}(t) \\
& +\alpha_{\omega}^{2} \mathbf{B}(\hat{\mathbf{s}}(t)-\mathbf{s}(t))-\eta_{\mathbf{m}} \sigma_{r} \alpha_{\omega} \mathbf{Q}(t) \mathbf{D}^{\top} \frac{d F_{\mathbf{w}}}{d \mathbf{u}^{\top}}(\hat{\mathbf{u}}(t)) \\
& -\eta_{\mathbf{m}} \mathbf{Q}(t) \mathbf{C}^{\top}(t)\left(\alpha_{\omega}^{2}(\hat{v}(t)-v(t))-r(t)-d(t)\right) .
\end{aligned}
$$

A bound on the solutions $\tilde{\mathbf{m}}(t)$ in (58) for time-varying inputs $\mathbf{u}(t)$ is presented in Lemma 22.
Lemma 22. For any $\epsilon_{0}, \epsilon_{1}, \epsilon_{3}, \epsilon_{5}, \epsilon_{\mathbf{z}} \in \mathbb{R}_{>0}$, any finite time $t_{1} \geq 0$, all $\alpha_{\omega} \leq \epsilon_{0}, \alpha_{\mathbf{z}} \leq \alpha_{\omega} \epsilon_{1} \leq \epsilon_{\mathbf{z}}, \eta_{\omega} \leq \alpha_{\mathbf{z}} \epsilon_{2}, \eta_{\mathbf{m}} \leq \eta_{\omega} \epsilon_{3}, \eta_{\mathbf{u}} \leq$ $\alpha_{\omega} \eta_{\mathbf{m}} \epsilon_{5}$, all $\lambda_{\mathbf{u}}, \sigma_{r} \in \mathbb{R}_{>0}$ and any $\delta_{\mathbf{z} 1}, \delta_{\mathbf{z} 2} \in \mathbb{R}_{\geq 0}$, the solutions $\overline{\tilde{\mathbf{m}}}$ are bounded for all $0 \leq t \leq t_{1}$, all $\tilde{\mathbf{u}}(0) \in \mathbb{R}^{n_{\mathbf{u}}}$, all $\tilde{\mathbf{z}}(0) \in \mathbb{R}^{n_{\mathbf{z}}}$, and all $\tilde{\mathbf{m}}(0) \in \mathbb{R}^{n_{\mathbf{u}}+1}$. In addition, for sufficiently small $\epsilon_{3}, \epsilon_{4}, \epsilon_{6} \in \mathbb{R}_{>0}$ and all $\alpha_{\omega} \lambda_{\mathbf{u}} \leq \eta_{\mathbf{m}} \epsilon_{4}$, there exist constants $c_{\mathbf{m} 1}, \ldots, c_{\mathbf{m} 8} \in \mathbb{R}_{>0}$ such that the solutions $\tilde{\mathbf{m}}$ satisfy

$$
\begin{aligned}
& \sup _{t \geq t_{1}}\|\tilde{\mathbf{m}}(t)\| \leq \sup _{t \geq t_{1}} \max \left\{c_{\mathbf{m} 1}\left\|\tilde{\mathbf{m}}\left(t_{1}\right)\right\|, c_{\mathbf{m} 2} \frac{\alpha_{\omega}^{2} \lambda_{\mathbf{u}}}{\eta_{\mathbf{m}}}\|\tilde{\mathbf{u}}(t)\|,\right. \\
& \alpha_{\omega}^{2} c_{\mathbf{m} 3}, c_{\mathbf{m} 4}\|\tilde{\mathbf{z}}(t)\|, \alpha_{\mathbf{z}} \delta_{\mathbf{z} 1} c_{\mathbf{m} 5}, \alpha_{\mathbf{z}} \delta_{\mathbf{z} 2} c_{\mathbf{m} 6}\|\tilde{\mathbf{u}}(t)\|^{2}, \\
& \left.\alpha_{\mathbf{z}} \alpha_{\omega}^{2} \delta_{\mathbf{z} 2} c_{\mathbf{m} 7}, \sqrt{\sigma_{r}} \alpha_{\omega} c_{\mathbf{m} 8}\|\tilde{\mathbf{u}}(t)\|\right\}, \\
& \limsup _{t \rightarrow \infty}\|\tilde{\mathbf{m}}(t)\| \leq \limsup _{t \rightarrow \infty} \max \left\{c_{\mathbf{m} 2} \frac{\alpha_{\omega}^{2} \lambda_{\mathbf{u}}}{\eta_{\mathbf{m}}}\|\tilde{\mathbf{u}}(t)\|,\right. \\
& \alpha_{\omega}^{2} c_{\mathbf{m} 3}, c_{\mathbf{m} 4}\|\tilde{\mathbf{z}}(t)\|, \alpha_{\mathbf{z}} \delta_{\mathbf{z} 1} c_{\mathbf{m} 5}, \alpha_{\mathbf{z}} \delta_{\mathbf{z} 2} c_{\mathbf{m} 6}\|\tilde{\mathbf{u}}(t)\|^{2}, \\
& \left.\alpha_{\mathbf{z}} \alpha_{\omega}^{2} \delta_{\mathbf{z} 2} c_{\mathbf{m} 7}, \sqrt{\sigma_{r}} \alpha_{\omega} c_{\mathbf{m} 8}\|\tilde{\mathbf{u}}(t)\|\right\},
\end{aligned}
$$

for all $t \geq t_{1}$, and all time-varying $\tilde{\mathbf{u}}(t) \in \mathbb{R}^{n_{\mathbf{u}}}$.

\section{Proof. See Appendix C.}

The dynamics of $\tilde{\mathbf{u}}, \tilde{\mathbf{z}}$, and $\tilde{\mathbf{m}}$ can be seen as feedback -interconnected subsystems for which the solutions satisfy the bounds in Lemmas 19,21 , and 22 , respectively. To verify that this feedback-interconnected system exhibits bounded solutions, the cyclic-small-gain criterion in Liu, Hill, and Jiang (2011) is employed. Let us consider arbitrary initial conditions $\mathbf{x}(0) \in \mathbb{R}^{n_{\mathbf{x}}}$, $\mathbf{Q}(0) \in \mathbb{R}^{n_{\mathbf{u}}+1 \times n_{\mathbf{u}}+1}$ symmetric and positive-definite, $\mathbf{z}(0) \in \mathbb{R}^{n_{\mathbf{z}}}$, $\hat{\mathbf{m}}(0) \in \mathbb{R}^{n_{\mathbf{u}}+1}$, an arbitrary large compact set $\mathcal{U}_{0} \subset \mathcal{U} \subset \mathbb{R}^{n_{\mathbf{u}}}$ of initial conditions for $\hat{\mathbf{u}}(t)$ with $\mathcal{U}_{0}:=\left\{\hat{\mathbf{u}} \in \mathbb{R}^{n_{\mathbf{u}}}:\|\tilde{\mathbf{u}}(t)\| \leq \rho_{\mathbf{u}}\right\}$ and with $\rho_{\mathbf{u}} \in \mathbb{R}_{>0}$, and $\mathcal{U}:=\left\{\hat{\mathbf{u}} \in \mathbb{R}^{n_{\mathbf{u}}}:\|\tilde{\mathbf{u}}(t)\| \leq L_{\mathbf{u}}\right\}$ a (arbitrarily large) compact set with some (sufficiently large) constant $L_{\mathbf{u}} \in \mathbb{R}_{>0}$. Later on, we will elaborate more on the role of the constant $L_{\mathbf{u}}$. For now, we consider it to exist, and additionally, we consider $\hat{\mathbf{u}}(t) \in \mathcal{U}$ for all time.

Under the conditions of Theorem 14, there exist sufficiently small constants $\epsilon_{0}, \ldots, \epsilon_{6} \in \mathbb{R}_{>0}$, such that the cyclic-small-gain criteria that follow from Lemmas 19, 21 and 22 and given by $c_{\mathbf{u} 1} c_{\mathbf{m} 2} \epsilon_{4}<1, c_{\mathbf{u} 1} c_{\mathbf{m} 6} \epsilon_{1} \delta_{\mathbf{z} 2} L_{\mathbf{u}}<1, c_{\mathbf{u} 1} c_{\mathbf{m} 4} c_{\mathbf{z} 4}\left(\epsilon_{0}, \epsilon_{1}, \epsilon_{3}, \epsilon_{5}, \epsilon_{\mathbf{z}}\right) \epsilon_{2}<$ $1, c_{\mathbf{u} 1} c_{\mathbf{m} 8} \sqrt{\epsilon_{6}}<1$ are satisfied, rendering the closed-loop system of the extended plant $\Sigma$ and the extremum-seeking controller to have bounded solutions. The final property that needs to be validated is that $\hat{\mathbf{u}}(t) \in \mathcal{U}$ for all time. We do this for the two temporal stages of convergence.

First, we consider $0 \leq t \leq t_{1}$. From the optimizer in (35) we have that $\|\dot{\tilde{\mathbf{u}}}(t)\| \leq \bar{\eta}_{\mathbf{u}}$. For any $\hat{\mathbf{u}}(0) \in \mathcal{U}_{0}$, it follows that $\sup _{0 \leq t \leq t_{1}}\|\tilde{\mathbf{u}}(t)\| \leq \rho_{\mathbf{u}}+\eta_{\mathbf{u}} t_{1}$. Under the conditions in Theorem 14, sufficiently small $\epsilon_{0}, \ldots, \epsilon_{6} \in \mathbb{R}_{>0}$ such that the cyclic-gain criteria are satisfied, and for any finite time $t_{1} \geq 0$, there exist tunable parameters $\eta_{\mathbf{u}}, \alpha_{\boldsymbol{\omega}}, \eta_{\mathbf{m}} \in \mathbb{R}_{>0}$ with $\eta_{\mathbf{u}} \leq \alpha_{\boldsymbol{\omega}} \eta_{\mathbf{m}} \epsilon_{5} \leq$ $\epsilon_{0}^{2} \epsilon_{1} \epsilon_{2} \epsilon_{3} \epsilon_{5}$, such that $\sup _{0 \leq t \leq t_{1}}\|\tilde{\mathbf{u}}(t)\| \leq \rho_{\mathbf{u}}+\eta_{\mathbf{u}} t_{1}<L_{\mathbf{u}}$ for sufficiently large $L_{\mathbf{u}} \in \mathbb{R}_{>0}$, implying $\hat{\mathbf{u}}(t) \in \mathcal{U}$ for $0 \leq t \leq t_{1}$.

Secondly, we consider $t \geq t_{1}$. From (49), (56), (59), and the satisfaction of the cyclic-small-gain criteria we obtain

$$
\begin{gathered}
\sup _{t \geq t_{1}}\|\tilde{\mathbf{u}}(t)\| \leq \max \left\{\left\|\tilde{\mathbf{u}}\left(t_{1}\right)\right\|, \frac{1}{\alpha_{\omega}} c_{\mathbf{u} 1} c_{\mathbf{m} 1}\left\|\tilde{\mathbf{m}}\left(t_{1}\right)\right\|,\right. \\
\alpha_{\omega} c_{\mathbf{u} 1} c_{\mathbf{m} 3}, \frac{1}{\alpha_{\omega}} c_{\mathbf{u} 1} c_{\mathbf{m} 4} c_{\mathbf{z} 1}\left\|\tilde{\mathbf{z}}\left(t_{1}\right)\right\|, \frac{\eta_{\omega}}{\alpha_{\mathbf{z}}} c_{\mathbf{z} 2} c_{\mathbf{u} 1} c_{\mathbf{m} 4}, \\
\left.\alpha_{\omega} c_{\mathbf{u} 1} c_{\mathbf{m} 4} c_{\mathbf{z} 3}, \frac{\alpha_{\mathbf{z}}}{\alpha_{\omega}} \delta_{\mathbf{z} 1} c_{\mathbf{u} 1} c_{\mathbf{m} 5}, \alpha_{\mathbf{z}} \alpha_{\omega} \delta_{\mathbf{z} 2} c_{\mathbf{u} 1} c_{\mathbf{m} 7}\right\},
\end{gathered}
$$

For the first term on the right-hand side of (61) we can make a similar observation as for the case when $0 \leq t \leq t_{1}$. Furthermore, for sufficiently small $\epsilon_{0}, \ldots, \epsilon_{6} \in \mathbb{R}_{>0}$, there exist tunable parameters $\alpha_{\omega}, \eta_{\omega}, \alpha_{\mathbf{z}}$ with $\alpha_{\omega} \leq \epsilon_{0}, \alpha_{\mathbf{z}} \leq \alpha_{\omega} \epsilon_{1}$ and $\eta_{\omega} \leq \alpha_{\mathbf{z}} \epsilon_{2}$, such that the third, and fifth through eight term on the right-hand side 
of (61) can be made arbitrarily small (smaller than $L_{\mathbf{u}}$ ). Finally, we can derive expressions for the second and fourth term on the right-hand side of (61) using Lemmas 21 and 22. Under the conditions of Theorem 14, for the initial conditions $\mathbf{x}(0), \mathbf{Q}(0), \mathbf{z}(0)$, $\hat{\mathbf{m}}(0)$, and $\hat{\mathbf{u}}(0) \in \mathcal{U}_{0}$, and sufficiently small constants $\epsilon_{0}, \ldots, \epsilon_{6} \in$ $\mathbb{R}_{>0}$, there exist tunable parameters $\alpha_{\mathbf{z}}, \alpha_{\boldsymbol{\omega}}, \eta_{\mathbf{u}}, \lambda_{\mathbf{u}}, \eta_{\mathbf{m}}, \eta_{\omega} \in \mathbb{R}_{>0}$ and $\sigma_{r} \in \mathbb{R}_{\geq 0}$ with $\alpha_{\omega} \leq \epsilon_{0}, \alpha_{\mathbf{z}} \leq \alpha_{\omega} \epsilon_{1}, \eta_{\omega} \leq \alpha_{\mathbf{z}} \epsilon_{2}, \eta_{\mathbf{m}} \leq$ $\eta_{\omega} \epsilon_{3}, \alpha_{\omega} \lambda_{\mathbf{u}} \leq \eta_{\mathbf{m}} \epsilon_{4}, \eta_{\mathbf{u}} \leq \alpha_{\omega} \eta_{\mathbf{m}} \epsilon_{5}$, and $\sigma_{r} \leq \epsilon_{6}$, such that the second and fourth term on the right-hand side of (61) can be made arbitrarily small (smaller than $L_{\mathbf{u}}$ ). As a result, $\hat{\mathbf{u}}(t) \in \mathcal{U}$ for all time.

Finally, from (50), (57), and (60) we obtain

$$
\begin{gathered}
\limsup _{t \rightarrow \infty}\|\tilde{\mathbf{u}}(t)\| \leq \max \left\{\alpha_{\omega} c_{\mathbf{u} 1} c_{\mathbf{m} 3}, \frac{\eta_{\omega}}{\alpha_{\mathbf{z}}} c_{\mathbf{z} 2} c_{\mathbf{u} 1} c_{\mathbf{m} 4},\right. \\
\left.\alpha_{\omega} c_{\mathbf{u} 1} c_{\mathbf{m} 4} c_{\mathbf{z} 3}, \frac{\alpha_{\mathbf{z}}}{\alpha_{\omega}} \delta_{\mathbf{z} 1} c_{\mathbf{u} 1} c_{\mathbf{m} 5}, \alpha_{\mathbf{z}} \alpha_{\omega} \delta_{\mathbf{z} 2} c_{\mathbf{u} 1} c_{\mathbf{m} 7}\right\} .
\end{gathered}
$$

Concluding, given a (time-varying) disturbance $\mathbf{w} \in \mathcal{W}$ and Assumptions 1, 5, 11, and 13. Consider arbitrary initial conditions $\mathbf{x}(0) \in \mathbb{R}^{n_{\mathbf{x}}}, \mathbf{Q}(0) \in \mathbb{R}^{n_{\mathbf{u}}+1 \times n_{\mathbf{u}}+1}$ symmetric and positive-definite, $\mathbf{z}(0) \in \mathbb{R}^{n_{\mathbf{z}}}, \hat{\mathbf{m}}(0) \in \mathbb{R}^{n_{\mathbf{u}}+1}$, and an arbitrary compact set $\mathcal{U}_{0} \subset$ $\mathbb{R}^{n_{\mathbf{u}}}$ of initial conditions for $\hat{\mathbf{u}}(t)$. There exist sufficiently small constants $\epsilon_{0}, \ldots, \epsilon_{6} \in \mathbb{R}_{>0}$, such that, for all tunable parameters $\alpha_{\mathbf{z}}, \alpha_{\boldsymbol{\omega}}, \eta_{\mathbf{u}}, \lambda_{\mathbf{u}}, \eta_{\mathbf{m}}, \eta_{\omega} \in \mathbb{R}_{>0}$ and $\sigma_{r} \in \mathbb{R}_{\geq 0}$ with $\alpha_{\boldsymbol{\omega}} \leq \epsilon_{0}, \alpha_{\mathbf{z}} \leq$ $\alpha_{\omega} \epsilon_{1}, \eta_{\omega} \leq \alpha_{\mathbf{z}} \epsilon_{2}, \eta_{\mathbf{m}} \leq \eta_{\omega} \epsilon_{3}, \alpha_{\omega} \lambda_{\mathbf{u}} \leq \eta_{\mathbf{m}} \epsilon_{4}, \quad \eta_{\mathbf{u}} \leq \alpha_{\omega} \eta_{\mathbf{m}} \epsilon_{5}$, and $\sigma_{r} \leq \epsilon_{6}$, the solutions of the closed-loop system are bounded. Moreover, the bound on the solutions $\hat{\mathbf{u}}(t)$ with $\hat{\mathbf{u}}(0) \in \mathcal{U}_{0}$ in (36) follows from (62) with $c_{1}:=\max \left\{c_{\mathbf{u} 1} c_{\mathbf{m} 3}, c_{\mathbf{u} 1} c_{\mathbf{m} 4} c_{\mathbf{z} 3}\right\}, c_{2}:=$ $c_{\mathbf{z} 2} c_{\mathbf{u} 1} c_{\mathbf{m} 4}, c_{3}:=c_{\mathbf{u} 1} c_{\mathbf{m} 5}, c_{4}:=c_{\mathbf{u} 1} c_{\mathbf{m} 7}$, which completes the proof of Theorem 14.

\section{Industrial case study}

In this section, we consider an industrial case study of the performance optimal tuning of a variable-gain controller (VGC) for a short-stroke wafer stage of a wafer scanner as also studied in Pavlov, Hunnekens, van de Wouw, and Nijmeijer (2013). Wafer scanners are part of the complex machinery used to manufacture integrated circuits (ICs). Fig. 4 illustrates some of the crucial elements of the wafer scanner. Basically, light, emitted by a laser, first passes through a so-called reticle which contains an image of the desired topology of an IC. The image is then projected onto a photo-sensitive layer on a so-called wafer by an optical system. The light in combination with the photo-sensitive layer induces a chemical reaction, resulting in the desired topology being transferred onto the wafer. A wafer typically contains 100 ICs, which requires extremely fast (re-)positioning of the wafer stage in three degrees-of-freedom with nm-accuracy.

Achieving nm-accuracy and high speeds of the wafer stage are realized by high-bandwidth linear controllers. However, due to the well-known waterbed-effect, see Freudenberg, Middleton, and Stefanpoulou (2000), increasing the bandwidth further to improve the ability to suppress low-frequency disturbances comes at the expense of an increased sensitivity to high-frequency disturbances and noise. Instead, a VGC can be used to balance this trade-off in a more desirable manner (Heertjes et al., 2009); it enables a higher bandwidth only when necessary. Although a VGC is intuitive in nature, performance optimal tuning of a VGC is far from trivial and heavily depends on the disturbance situation at hand. In this section, we employ the ESC approach proposed in Section 2 to tune the VGC for optimal steady-state performance of the wafer stage, illustrating the effectiveness of the proposed ESC approach.

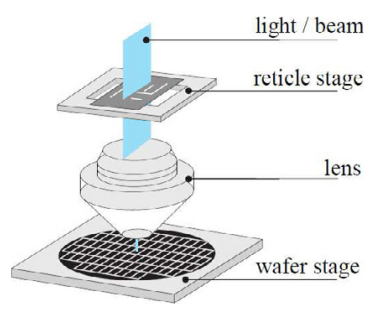

Fig. 4. Schematic overview of the crucial elements of the wafer scanner.

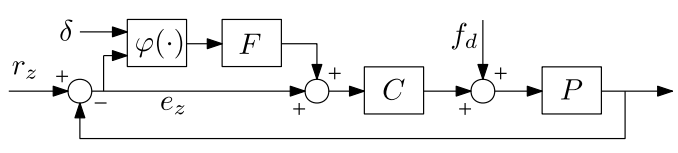

Fig. 5. The closed-loop variable-gain control scheme.

\subsection{Variable-gain controlled motion stage}

The VGC structure is shown in Fig. 5. The scheme consists of a plant $P$, representing the dynamics of a short-stroke wafer stage in $z$-direction, and a nominal linear controller $C$, having transfer functions $P(s)$ and $C(s)$, respectively, with $s \in \mathbb{C}$ being the Laplacian variable, (time-varying) force disturbances $f_{d}(t)$, a nonlinear control element $\varphi(\cdot, \cdot)$, and a shaping filter $F(s)$. Furthermore, $r_{z}$ denotes the setpoint in $z$-direction, and $e_{z}$ denotes the tracking error in $z$-direction, which is the performance variable to-be optimized. The setpoint is $r_{z}=0$. The nonlinearity $\varphi\left(e_{z}, \delta\right)$, representing the variable-gain element with $e_{z}$ as input, is given by a dead-zone characteristic

$\varphi\left(e_{z}, \delta\right)= \begin{cases}\alpha\left(e_{z}+\delta\right), & \text { if } e_{z}<-\delta, \\ 0, & \text { if }\left|e_{z}\right| \leq \delta, \\ \alpha\left(e_{z}-\delta\right), & \text { if } e_{z}>\delta,\end{cases}$

where $\alpha$ and $\delta$ denote the additional gain and the dead-zone length, respectively. The plant is modeled as a 4 th-order massspring-damper-mass system, admitting the following transfer function representation

$P(s)=\frac{m_{1} s^{2}+b s+k}{s^{2}\left(m_{1} m_{2} s^{2}+b\left(m_{1}+m_{2}\right) s+k\left(m_{1}+m_{2}\right)\right)}$,

with the following numerical values: $m_{1}=5 \mathrm{~kg}, m_{2}=17.5 \mathrm{~kg}$, $k=7.5 \cdot 10^{7} \mathrm{~N} / \mathrm{m}, b=90 \mathrm{~N} \mathrm{~s} / \mathrm{m}$. The nominal, and stabilizing linear controller consists of a PID-controller $C_{p i d}$, a second-order low-pass filter $C_{l p}$ and a notch filter $C_{n}$, i.e. $C(s)=C_{p i d}(s) C_{l p}(s) C_{n}(s)$. The filters are given by $C_{p i d}(s)=\left(k_{p}\left(s^{2}+\left(\omega_{i}+\omega_{d}\right) s+\omega_{i} \omega_{d}\right)\right) /\left(\omega_{d} s\right)$, where $k_{p}=6.9 \cdot 10^{6} \mathrm{~N} / \mathrm{m}, \omega_{d}=3.8 \cdot 10^{2} \mathrm{rad} / \mathrm{s}$, and $\omega_{i}=3.14 \cdot 10^{2}$ $\mathrm{rad} / \mathrm{s} ; C_{l p}(s)=\omega_{l p}^{2} /\left(\mathrm{s}^{2}+2 \beta_{l p} \omega_{l p} s+\omega_{l p}^{2}\right)$, where $\omega_{l p}=3.04 \cdot 10^{3}$ $\mathrm{rad} / \mathrm{s}$, and $\beta_{p}=0.08 ; C_{n}(s)=\left(s^{2}+2 \beta_{z} \omega_{z} s+\omega_{z}^{2}\right) /\left(s^{2}+2 \beta_{p} \omega_{p} s+\omega_{p}^{2}\right)$, where $\omega_{z}=4.39 \cdot 10^{3} \mathrm{rad} / \mathrm{s}, \omega_{p}=5.03 \cdot 10^{3} \mathrm{rad} / \mathrm{s}, \beta_{z}=$ $2.7 \cdot 10^{-3}$, and $\beta_{p}=0.88$. The shaping filter $F(s)$ is given by $F(s)=\left(s^{2}+2 \beta_{z, F} \omega_{z, F} s+\omega_{z, F}^{2}\right) /\left(s^{2}+2 \beta_{p, F} \omega_{p, F} s+\omega_{p, F}^{2}\right)$, with $\omega_{z, F}=\omega_{p, F}=2 \cdot 10^{3} \mathrm{rad} / \mathrm{s}, \beta_{z, F}=0.6, \beta_{p, F}=4.8$.

The disturbance $f_{d}(t)$ consists of a low-frequency force disturbance contribution induced by ( $3^{\text {rd }}$-order) setpoint accelerations in the $x$-and $y$-direction of the wafer stage (see Fig. 6 for a scaled acceleration profile in $y$-direction), and a high-frequency force disturbance contribution, modeled as a signal containing multiple sinusoidal components with both random frequencies between 200-500 Hz and phases.

From Theorem 1 in Pavlov et al. (2013), which is based on circle criterion type arguments, it can be concluded that if the additional gain is chosen as $\alpha \leq 4.34$, then the closed-loop system satisfies Assumption 5. The dead-zone length $\delta$ turns out to be a 


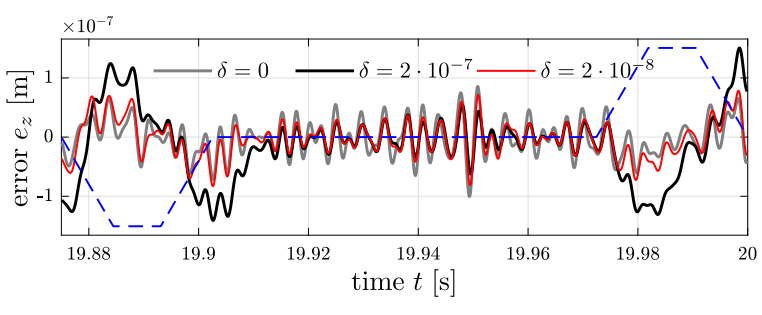

Fig. 6. The steady-state tracking error for three cases with additional gain $\alpha=4$.33; (i) $\delta=2 \cdot 10^{-7}$, referred to as the nominal low-gain linear controller, (ii) $\delta=0$, referred to as the high-gain linear controller, and (iii) $\delta=2 \cdot 10^{-8}$, in which the variable-gain controller is employed. The blue line is a scaled acceleration profile in $y$-direction.

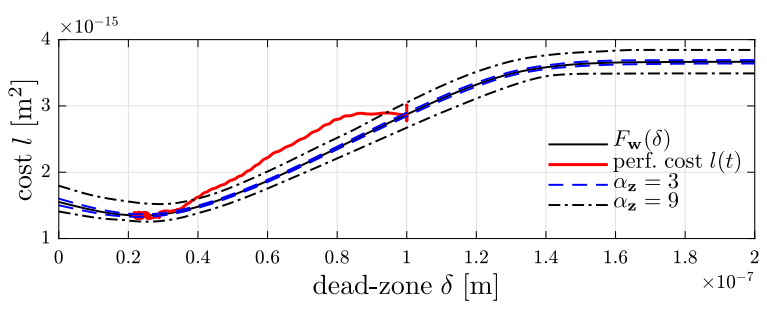

Fig. 7. Objective function $F_{\mathbf{w}}$, bounds on $F_{\mathbf{w}}$ for $\alpha_{\mathbf{z}}=3$, and $\alpha_{\mathbf{z}}=9$ that illustrate the bound in (16) in Assumption 11, and the minimization of the performance cost $l(t)$ by adaptation of $\delta$.
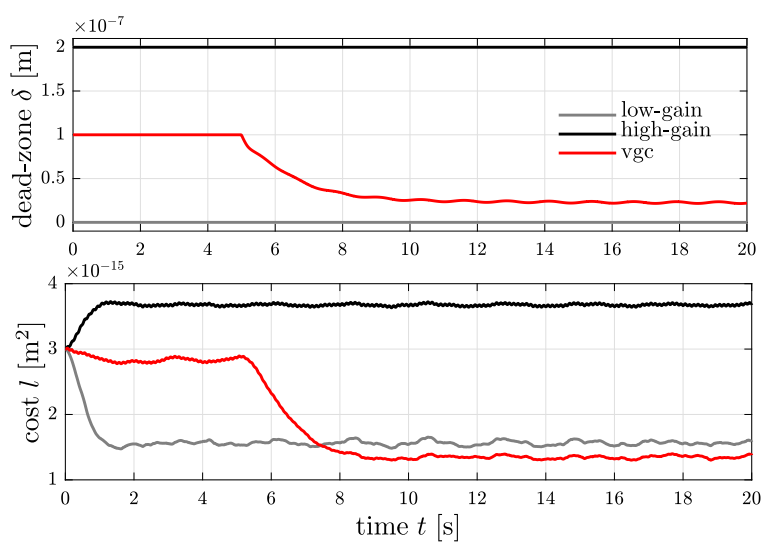

Fig. 8. Convergence of the tunable parameter $\delta$ towards $\delta^{*}$ and performance cost in case of two constant values of $\delta$, associated with (i) the nominal low-gain and (ii) the high-gain controller.

stability-invariant tunable plant parameter; however, the choice for $\delta$ does affect significantly the achievable tracking performance as the optimal value for $\delta$ depends heavily on the disturbance situation at hand, and is typically chosen in a heuristic manner. Fig. 6 shows a time interval of the tracking error for three cases with additional gain $\alpha=4.33$; (i) $\delta=2 \cdot 10^{-7}$, referred to as the nominal low-gain linear controller, (ii) $\delta=0$, referred to as the high-gain linear controller, and (iii) $\delta=2 \cdot 10^{-8}$, in which the VGC is employed. As such, we propose to tune the dead-zone length $\delta$ in real-time by the ESC scheme presented in Sections 2 and 3 to optimize the tracking performance.

\subsection{Optimization using extremum-seeking control}

For the ESC scheme as presented in Sections 2 and 3, the measured performance variable is the tracking error $e_{z}$, and the tunable system parameter is the dead-zone length $\delta$. We choose the cost function as $Z\left(e_{z}(t)\right)=e_{z}^{2}(t)$, and the filter $\Sigma_{f}$ as a
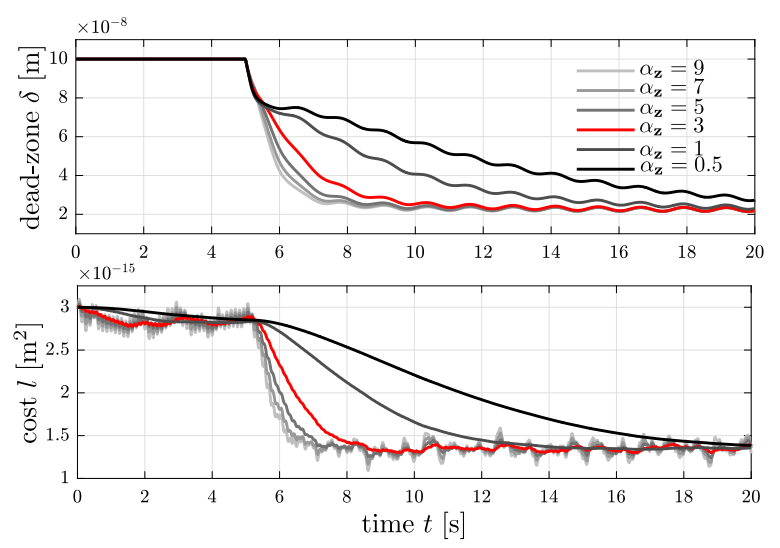

Fig. 9. Convergence of the tunable parameter $\delta$ towards $\delta^{*}$ and the associated performance cost $l$ for different values for $\alpha_{\mathbf{z}}$.

second-order low-pass filter admitting the following state-space formulation

$\Sigma_{f}:\left\{\begin{aligned} \dot{z}_{1}(t) & =\alpha_{\mathbf{z}} z_{2}(t) \\ \dot{z}_{2}(t) & =\alpha_{\mathbf{z}}\left(y(t)-2 \beta_{\mathbf{z}} z_{2}(t)-z_{1}(t)\right) \\ l(t) & =z_{1}(t)\end{aligned}\right.$

which is of the form in (9). Furthermore, the specific choice of the cost function $Z$ and filter $\Sigma_{f}$ with $\alpha_{\mathbf{z}}, \beta_{\mathbf{z}} \in \mathbb{R}_{>0}$, renders the satisfaction of (10), (12), and Property 8 . The objective function is depicted in Fig. 7. Moreover, bounds on $F_{\mathbf{w}}$ for $\alpha_{\mathbf{z}}=3$, and $\alpha_{\mathbf{z}}=9$ are shown in Fig. 7 that illustrate the bound in (16) from Assumption 11. Clearly, for smaller values of $\alpha_{\mathbf{z}}$ and fixed $\delta$, the resulting (steady-state) output $l(t)$ is a better approximation of the steady-state performance $F_{\mathbf{w}}(\delta)$. Clearly, from the objective function it can be observed that the optimal dead-zone length $\delta^{*}$ for this specific disturbance situation is approximately $2 \cdot 10^{-8}$. The parameters of the extremum-seeking controller are chosen as $\beta_{\mathbf{z}}=\frac{1}{2} \sqrt{2}, \alpha_{\mathbf{z}}=3, \eta_{\omega}=5, \alpha_{\omega}=1 \cdot 10^{-9}, \eta_{\mathbf{m}}=0.05, \sigma_{r}=1 \cdot 10^{-6}$, $\lambda_{\mathbf{u}}=1 \cdot 10^{10}, \hat{H}(t, \delta)=0.55$ for all $t$ and $\delta$, and $\eta_{\mathbf{u}}=1$. The initial conditions are chosen as $\mathbf{z}^{\top}(0)=\left[\begin{array}{ll}3 \cdot 10^{-15} & 1 \cdot 10^{-17}\end{array}\right]$, $\hat{\mathbf{m}}^{\top}(0)=\left[\begin{array}{ll}3 \cdot 10^{-15} & 0\end{array}\right], \mathbf{Q}(0)=\left[\begin{array}{cc}1 & 0 \\ 0 & \frac{2}{1+2 \sigma_{r}}\end{array}\right]$ and $\hat{\delta}(0)=1 \cdot 10^{-7}$. The extremum-seeking controller is enabled at $t=5$ seconds. Fig. 8 shows the dead-zone length $\delta$ and the measured performance cost $l(t)$ as a function of time for three cases; cases 1 and 2 in which two constant values for $\delta$ are used, namely $\delta=2 \cdot 10^{-7}$ and $\delta=0$, and case 3 in which $\delta$ is tuned by an extremum-seeking controller. It can be seen that the plant parameter $\delta$ and the corresponding performance cost $l$ converge to the performance optimal region, as illustrated in Fig. 7. Fig. 6 shows the measured tracking error for the low-gain, high-gain, and optimally tuned variable-gain controller.

In Fig. 9 the effect of the additional time-scale induced by the additional filter $\Sigma_{f}$ is shown for different values for $\alpha_{\mathbf{z}}$. Basically, for smaller values of $\alpha_{\mathbf{z}}$ the transient response of the extended plant is slower, resulting in a slower convergence to the optimal setting. However, we suffer less from time-varying behavior. Decreasing the value of $\alpha_{\mathbf{z}}$ further will eventually lead to loss of time-scale separation. For larger values of $\alpha_{\mathbf{z}}$, the transient response is much faster, and thus the convergence to the optimal setting $\delta^{*}$ is faster. However, for even larger values of $\alpha_{\mathbf{z}}$ convergence to the optimal setting is not guaranteed; in such a case the extremum-seeking controller suffers from the time-varying behavior and is not able to estimate a proper gradient. 


\section{Conclusion}

In this work, we have developed an ESC approach for optimization of time-varying steady-state responses of general nonlinear systems. The proposed ESC method employs a so-called dynamic cost function. This dynamic cost function allows for the characterization of a static performance map, despite the presence of time-varying disturbances which induces time-varying steady-state responses. We have proven that the proposed ESC method achieves semi-global practical asymptotic stability of the closed-loop scheme in the presence of bounded and time-varying disturbances. An illustrative example is provided that shows the steady-state performance optimization of a closed-loop variablegain controlled motion system subject to a time-varying force disturbance by means of the proposed ESC approach.

\section{Acknowledgments}

This work is part of the research programme CHAMeleon with project number 13896, which is (partly) financed by the Netherlands Organisation for Scientific Research (NWO), The Netherlands. Mark Haring was with the Centre for Autonomous Marine Operations and Systems, Department of Engineering Cybernetics, NTNU, Norwegian University of Science and Technology, 7491 Trondheim, Norway. The work of Mark Haring is sponsored by the Research Council of Norway with project number 250725, and through the Centres of Excellence, Norway funding scheme, project number 223254 - Centre for Autonomous Marine Operations and Systems (NTNU-AMOS).

\section{Appendix A. Proof of Lemma 17}

By using the function $V_{\mathbf{x}}$ in Lemma 16 as a Lyapunov function candidate for the $\tilde{\mathbf{x}}$-dynamics in (44) with time-varying inputs $\mathbf{u}(t)$ and generated by (23) and (35), we obtain the following expression for $\dot{V}_{\mathbf{x}}$ :

$\dot{V}_{\mathbf{x}}=\frac{\partial V_{\mathbf{x}}}{\partial t}+\frac{\partial V_{\mathbf{x}}}{\partial \tilde{\mathbf{x}}} \tilde{\mathbf{f}}_{\mathbf{w}}(t, \tilde{\mathbf{x}}, \mathbf{u})+\left(\frac{\partial V_{\mathbf{x}}}{\partial \mathbf{u}}-\frac{\partial V_{\mathbf{x}}}{\partial \tilde{\mathbf{x}}} \frac{\partial \overline{\mathbf{x}}_{\mathbf{w}}}{\partial \mathbf{u}}\right) \dot{\mathbf{u}}$,

for all $\tilde{\mathbf{x}} \in \mathbb{R}^{n_{\mathbf{x}}}$, all time-varying inputs $\mathbf{u}(t) \in \mathbb{R}^{n_{\mathbf{u}}}$, and all $t$. Note that in (A.1) we have omitted most arguments for notational clarity. Using Assumption 1, (5) in Assumption 5, the inequalities from Lemma 16, and Young's inequality, it follows that

$\dot{V}_{\mathbf{x}} \leq-\frac{\gamma_{\mathbf{x} 3}}{2 \gamma_{\mathbf{x} 2}} V_{\mathbf{x}}(t, \tilde{\mathbf{x}}, \mathbf{u})+\frac{\left(\gamma_{\mathbf{x} 4} L_{\mathbf{x u}}+\gamma_{\mathbf{x} 5}\right)^{2}}{2 \gamma_{\mathbf{x} 3}}\|\dot{\mathbf{u}}\|^{2}$.

To find an upperbound for $\|\dot{\mathbf{u}}\|$, it follows from (23) that $\dot{\mathbf{u}}=$ $\dot{\hat{\mathbf{u}}}+\alpha_{\boldsymbol{\omega}} \dot{\boldsymbol{\omega}}$. From (24), we have that there exists a constant $L_{\omega 1} \in$ $\mathbb{R}_{>0}$ such that $\|\dot{\boldsymbol{\omega}}\| \leq \eta_{\omega} L_{\omega 1}$. Furthermore, from (35) we have that $\|\dot{\hat{\mathbf{u}}}\| \leq \eta_{\mathbf{u}}$. Therefore, we obtain an upperbound on $\|\dot{\mathbf{u}}\|$ which is given by

$\|\dot{\mathbf{u}}\| \leq \alpha_{\omega} \eta_{\omega}\left(\epsilon_{3} \epsilon_{5}+L_{\omega 1}\right)$

for all $\epsilon_{3}, \epsilon_{5} \in \mathbb{R}_{>0}$, all $\eta_{\mathbf{u}} \leq \alpha_{\omega} \eta_{\mathbf{m}} \epsilon_{5}$, and all $\eta_{\mathbf{m}} \leq \eta_{\omega} \epsilon_{3}$ (as guaranteed by the conditions in Theorem 14). Substitution in (A.2), and applying the comparison lemma (Khalil, 2002, Lemma 3.4), it follows that

$$
\begin{gathered}
V_{\mathbf{x}}(t, \tilde{\mathbf{x}}(t), \mathbf{u}(t)) \leq V_{\mathbf{x}}(0, \tilde{\mathbf{x}}(0), \mathbf{u}(0)) e^{-\frac{\gamma_{\mathbf{x} 3}}{2 \gamma_{\mathbf{x} 2}} t} \\
+\alpha_{\omega}^{2} \eta_{\omega}^{2} \gamma_{\mathbf{x} 2} \frac{\left(\gamma_{\mathbf{x} 4} L_{\mathbf{x} \mathbf{u}}+\gamma_{\mathbf{x} 5}\right)^{2}}{\gamma_{\mathbf{x} 3}^{2}}\left(\epsilon_{3} \epsilon_{5}+L_{\omega 1}\right)^{2},
\end{gathered}
$$

for all $t \geq 0$, all $\tilde{\mathbf{x}}(0) \in \mathbb{R}^{n_{\mathbf{x}}}$, and all time-varying $\mathbf{u}(t) \in \mathbb{R}^{n_{\mathbf{u}}}$. From (41) in Lemma 16 , we obtain

$\|\tilde{\mathbf{x}}(t)\| \leq \max \left\{c_{\mathbf{x} 1}\|\tilde{\mathbf{x}}(0)\| e^{-\beta_{\mathbf{x}} t}, \alpha_{\omega} \eta_{\omega} c_{\mathbf{x} 2}\right\}$,

with positive constants $\beta_{\mathbf{x}}=\frac{\gamma_{\mathbf{x} 3}}{4 \gamma_{\mathbf{x} 2}}, c_{\mathbf{x} 1}=\sqrt{\frac{2 \gamma_{\mathbf{x} 2}}{\gamma_{\mathbf{x} 1}}}$, and $c_{\mathbf{x} 2}=$ $\sqrt{\frac{2 \gamma_{\mathbf{x} 2}}{\gamma_{\mathbf{x} 1}}} \frac{\gamma_{\mathbf{x} 4} L_{\mathbf{x u}}+\gamma_{\mathbf{x} 5}}{\gamma_{\mathbf{x} 3}}\left(\epsilon_{3} \epsilon_{5}+L_{\omega 1}\right)$, which completes the proof of

\section{Appendix B. Proof of Lemma 21}

By using the function $V_{\mathbf{z}}$ in Lemma 20 as a Lyapunov function candidate for the $\tilde{\mathbf{z}}$-dynamics in (55) with time-varying inputs $\mathbf{u}(t)$ and generated by (23) and (35), we obtain the following expression for $\dot{V}_{\mathbf{z}}$ :

$$
\begin{aligned}
\dot{V}_{\mathbf{z}}= & \frac{\partial V_{\mathbf{z}}}{\partial t}+\alpha_{\mathbf{z}} \frac{\partial V_{\mathbf{z}}}{\partial \tilde{\mathbf{z}}} \tilde{\mathbf{h}}_{\mathbf{w}}\left(t, \tilde{\mathbf{z}}, \mathbf{u}, \alpha_{\mathbf{z}}\right) \\
& +\alpha_{\mathbf{z}} \frac{\partial V_{\mathbf{z}}}{\partial \tilde{\mathbf{z}}}\left(\mathbf{h}(\mathbf{z}, y)-\mathbf{h}\left(\mathbf{z}, \bar{y}_{\mathbf{w}}(t, \mathbf{u})\right)\right) \\
& +\left(\frac{\partial V_{\mathbf{z}}}{\partial \mathbf{u}}-\frac{\partial V_{\mathbf{z}}}{\partial \tilde{\mathbf{z}}}\left(\frac{\partial \overline{\mathbf{z}}_{\mathbf{w}}}{\partial \mathbf{u}}-\frac{d \mathbf{q}_{\mathbf{w}}}{d \mathbf{u}}+\frac{d \mathbf{q}_{\mathbf{w}}}{d \mathbf{u}}\right)\right) \dot{\mathbf{u}},
\end{aligned}
$$

for all $\tilde{\mathbf{z}} \in \mathbb{R}^{n_{\mathbf{z}}}$, all time-varying inputs $\mathbf{u}(t) \in \mathbb{R}^{n_{\mathbf{u}}}$, for all $t$, and all $y, \bar{y}_{\mathbf{w}} \in \mathbb{R}$ satisfying (8) and (11), respectively. Note that in (B.1) we have omitted most arguments for notational clarity. Using the inequalities from Lemma 20 and Assumption 11, it follows that

$$
\begin{aligned}
\dot{V}_{\mathbf{z}} \leq & -\alpha_{\mathbf{z}} \gamma_{\mathbf{z} 3}\|\tilde{\mathbf{z}}\|^{2}+\alpha_{\mathbf{z}} \gamma_{\mathbf{z} 4} L_{\mathbf{z} 1}\|\tilde{\mathbf{z}}\|\|\dot{\mathbf{u}}\| \\
& +\alpha_{\mathbf{z}} \gamma_{\mathbf{z} 4}\|\tilde{\mathbf{z}}\|\left\|\mathbf{h}(\mathbf{z}, y)-\mathbf{h}\left(\mathbf{z}, \bar{y}_{\mathbf{w}}(t, \mathbf{u})\right)\right\|+\gamma_{\mathbf{z} 5}\|\tilde{\mathbf{z}}\|\|\dot{\mathbf{u}}\| \\
& +\left(\gamma_{\mathbf{z} 6}+\alpha_{\mathbf{z}} \gamma_{\mathbf{z} 4} L_{\mathbf{z} 2}+\gamma_{\mathbf{z} 4} L_{\mathbf{q}}\right)\left\|\mathbf{u}-\mathbf{u}_{\mathbf{w}}^{*}\right\|\|\tilde{\mathbf{z}}\|\|\dot{\mathbf{u}}\| .
\end{aligned}
$$

By defining $\tilde{y}:=y-\bar{y}_{\mathbf{w}}(t, \mathbf{u})$ and the bounds in (12), it follows that

$$
\left\|\mathbf{h}(\mathbf{z}, y)-\mathbf{h}\left(\mathbf{z}, \bar{y}_{\mathbf{w}}(t, \mathbf{u})\right)\right\| \leq L_{\mathbf{h} y}\|\tilde{y}\| .
$$

By defining $\tilde{\mathbf{e}}:=\mathbf{e}-\overline{\mathbf{e}}_{\mathbf{w}}(t, \mathbf{u})$, using (8), (11), and the bounds in (10), it follows that

$$
\begin{aligned}
\|\tilde{y}\| \leq & L_{Z \mathbf{e}}\left\|\overline{\mathbf{e}}_{\mathbf{w}}(t, \mathbf{u})-\overline{\mathbf{e}}_{\mathbf{w}}\left(t, \mathbf{u}_{\mathbf{w}}^{*}\right)\right\|\|\tilde{\mathbf{e}}\|+L_{Z *}\|\tilde{\mathbf{e}}\| \\
& +\frac{1}{2} L_{Z \mathbf{e}}\|\tilde{\mathbf{e}}\|^{2}+L_{Z \mathbf{u}}\left\|\mathbf{u}-\mathbf{u}_{\mathbf{w}}^{*}\right\|\|\tilde{\mathbf{e}}\|,
\end{aligned}
$$

with $L_{Z *}=\left\|\frac{\partial Z}{\partial \mathbf{e}}\left(\overline{\mathbf{e}}_{\mathbf{w}}\left(t, \mathbf{u}_{\mathbf{w}}^{*}\right), \mathbf{u}_{\mathbf{w}}^{*}\right)\right\| \in \mathbb{R}_{>0}$. From Assumption 1 and (5) in Assumption 5, it follows that

$$
\begin{aligned}
& \|\tilde{\mathbf{e}}\|=\left\|\mathbf{g}(\mathbf{x}, \mathbf{u}, \mathbf{w})-\mathbf{g}\left(\overline{\mathbf{x}}_{\mathbf{w}}(t, \mathbf{u}), \mathbf{u}, \mathbf{w}\right)\right\| \leq L_{\mathbf{g x}}\|\tilde{\mathbf{x}}\|, \\
& \left\|\overline{\mathbf{e}}_{\mathbf{w}}(t, \mathbf{u})-\overline{\mathbf{e}}_{\mathbf{w}}\left(t, \mathbf{u}_{\mathbf{w}}^{*}\right)\right\| \leq\left(L_{\mathbf{g u}}+L_{\mathbf{g} \mathbf{x}} L_{\mathbf{x} \mathbf{u}}\right)\left\|\mathbf{u}-\mathbf{u}_{\mathbf{w}}^{*}\right\| .
\end{aligned}
$$

From (24), it follows that there exists a constant $L_{\omega 2} \in \mathbb{R}_{>0}$ such that $\|\omega\| \leq L_{\omega 2}$. From this fact, (23), and the coordinate transformation in (37), it follows that

$$
\left\|\mathbf{u}-\mathbf{u}_{\mathbf{w}}^{*}\right\| \leq\|\tilde{\mathbf{u}}\|+\alpha_{\omega} L_{\omega 2}
$$

Substitution of (B.3) to (B.7) into (B.2), applying Young's inequality, using the bound on $\|\dot{\mathbf{u}}\|$ in (A.3), and the inequality in (51) from Lemma 20, results in

$$
\begin{aligned}
\dot{V}_{\mathbf{z}} \leq & -\frac{\alpha_{\mathbf{z}} \gamma_{\mathbf{z} 3}}{2 \gamma_{\mathbf{z} 2}} V_{\mathbf{z}}\left(t, \tilde{\mathbf{z}}, \mathbf{u}, \alpha_{\mathbf{z}}\right)+\frac{9 \alpha_{\mathbf{z}} z_{1}^{2}}{2 \gamma_{\mathbf{z} 3}}\|\tilde{\mathbf{x}}\|^{4} \\
& +\frac{9 \alpha_{\mathbf{z}} z_{8}^{2}}{2 \gamma_{\mathbf{z} 3}}\|\tilde{\mathbf{x}}\|^{2}+\frac{9 \alpha_{\mathbf{z}} \alpha_{\omega}^{2} z_{9}^{2}}{2 \gamma_{\mathbf{z} 3}}\|\tilde{\mathbf{x}}\|^{2}+\frac{9 \alpha_{\mathbf{z}} z_{2}^{2}}{2 \gamma_{\mathbf{z} 3}}\|\tilde{\mathbf{u}}\|^{2}\|\tilde{\mathbf{x}}\|^{2} \\
& +\frac{9 \alpha_{\omega}^{2} \eta_{\omega}^{2}}{2 \alpha_{\mathbf{z}} \gamma_{\mathbf{z} 3}} z_{10}^{2}\left(\epsilon_{0} \epsilon_{1} z_{4}+z_{3}\right)^{2}\|\tilde{\mathbf{u}}\|^{2} \\
& +\frac{9 \alpha_{\omega}^{2} z_{5}^{2}}{2 \alpha_{\mathbf{z}} \gamma_{\mathbf{z} 3}} \alpha_{\omega}^{2} \eta_{\omega}^{2} z_{10}^{2}+\frac{9 \alpha_{\omega}^{2} \eta_{\omega}^{2}}{2 \alpha_{\mathbf{z}} \gamma_{\mathbf{z} 3}} z_{10}^{2}\left(\epsilon_{0} \epsilon_{1} z_{6}+z_{7}\right)^{2},
\end{aligned}
$$

for all $\alpha_{\omega} \leq \epsilon_{0}$, all $\alpha_{\mathbf{z}} \leq \alpha_{\omega} \epsilon_{1}$, all $\eta_{\mathbf{m}} \leq \eta_{\omega} \epsilon_{3}$, and all $\eta_{\mathbf{u}} \leq \alpha_{\omega} \eta_{\mathbf{m}} \epsilon_{5}$, where we have used that $\epsilon_{h}^{2} \epsilon_{i}^{2} z_{j}^{2}+z_{k}^{2} \leq\left(\epsilon_{h} \epsilon_{i} z_{j}+z_{k}\right)^{2}$ when $\epsilon_{h}, \epsilon_{i}, z_{j}, z_{k} \in \mathbb{R}_{>0}$, and with $z_{10}=\epsilon_{3} \epsilon_{5}+L_{\omega 1}$, and some positive constants $z_{1}, \ldots, z_{9} \in \mathbb{R}_{>0}$. From Lemmas 17 and 19, it follows that, for any finite time $t_{1} \geq 0$, the solutions $\tilde{\mathbf{x}}(t)$ and $\tilde{\mathbf{u}}(t)$ are bounded for all $0 \leq t \leq t_{1}$. As such, the right-hand side of (B.8) is bounded for all $0 \leq t \leq t_{1}$. Therefore, $V_{\mathbf{z}}\left(t, \tilde{\mathbf{z}}(t), \tilde{\mathbf{u}}(t), \alpha_{\mathbf{z}}\right)$ is bounded for all $0 \leq t \leq t_{1}$, and from the bounds in Lemma 20 it follows that the solutions $\tilde{\mathbf{z}}(t)$ are bounded for all $0 \leq t \leq t_{1}$.

From Lemma 17, it follows that here exists a time instance $t_{1} \geq 0$, such that $\|\tilde{\mathbf{x}}\| \leq \alpha_{\omega} \eta_{\omega} c_{\mathbf{x} 2}$. Substitution in (B.8), subsequently applying the comparison lemma and using the inequality 
(51) in Lemma 20, we obtain the bound on $\tilde{\mathbf{z}}$ as

$$
\begin{aligned}
& \sup _{t \geq t_{1}}\|\tilde{\mathbf{z}}(t)\| \leq \frac{6}{\gamma_{\mathbf{z} 3}} \sqrt{\frac{\gamma_{\mathbf{z} 2}}{\gamma_{\mathbf{z} 1}}} \sup _{t \geq t_{1}} \max \left\{\left\|\tilde{\mathbf{z}}\left(t_{1}\right)\right\|,\right. \\
& \quad \frac{\alpha_{\omega} \eta_{\omega}}{\alpha_{\mathbf{z}}}\left(z_{10}\left(\epsilon_{0} \epsilon_{1} z_{6}+z_{7}\right)+\epsilon_{0} \epsilon_{1} z_{8} c_{\mathbf{x} 2}\right), \\
& \alpha_{\omega}^{2} \epsilon_{2}\left(z_{10} z_{5}+\epsilon_{0} \epsilon_{1} c_{\mathbf{x} 2}\left(z_{9}+\epsilon_{0} \epsilon_{1} \epsilon_{2} z_{1} c_{\mathbf{x} 2}\right)\right), \\
& \left.\quad \frac{\alpha_{\omega} \eta_{\omega}}{\alpha_{\mathbf{z}}}\left(z_{10}\left(\epsilon_{0} \epsilon_{1} z_{4}+z_{3}\right)+\epsilon_{0} \epsilon_{1} z_{2} c_{\mathbf{x} 2}\right)\|\tilde{\mathbf{u}}(t)\|\right\}, \\
& \limsup _{t \rightarrow \infty}\|\tilde{\mathbf{z}}(t)\| \leq \frac{6}{\gamma_{\mathbf{z} 3}} \sqrt{\frac{\gamma_{\mathbf{z} 2}}{\gamma_{\mathbf{z} 1}}} \limsup _{t \rightarrow \infty} \max \{ \\
& \frac{\alpha_{\omega} \eta_{\omega}}{\alpha_{\mathbf{z}}}\left(z_{10}\left(\epsilon_{0} \epsilon_{1} z_{6}+z_{7}\right)+\epsilon_{0} \epsilon_{1} z_{8} c_{\mathbf{x} 2}\right), \\
& \alpha_{\omega}^{2} \epsilon_{2}\left(z_{10} z_{5}+\epsilon_{0} \epsilon_{1} c_{\mathbf{x} 2}\left(z_{9}+\epsilon_{0} \epsilon_{1} \epsilon_{2} z_{1} c_{\mathbf{x} 2}\right)\right), \\
& \left.\frac{\alpha_{\omega} \eta_{\omega}}{\alpha_{\mathbf{z}}}\left(z_{10}\left(\epsilon_{0} \epsilon_{1} z_{4}+z_{3}\right)+\epsilon_{0} \epsilon_{1} z_{2} c_{\mathbf{x} 2}\right)\|\tilde{\mathbf{u}}(t)\|\right\} .
\end{aligned}
$$

for all $t \geq t_{1}$, all $\alpha_{\omega} \leq \epsilon_{0}$, all $\alpha_{\mathbf{z}} \leq \alpha_{\omega} \epsilon_{1}$, all $\eta_{\omega} \leq \alpha_{\mathbf{z}} \epsilon_{2}$, all $\eta_{\mathbf{m}} \leq \eta_{\omega} \epsilon_{3}$, and all $\eta_{\mathbf{u}} \leq \alpha_{\omega} \eta_{\mathbf{m}} \epsilon_{5}$. The bounds in (56) and (57) of Lemma 21 follow from (B.9) and (B.10), respectively, which completes the proof of Lemma 21.

\section{Appendix C. Proof of Lemma 22}

The proof of Lemma 22 builds on the proof of Lemma 2.13 in Haring (2016, Chapter 2). We define the following Lyapunov function candidate for the $\tilde{\mathbf{m}}$-dynamics in (58):

$V_{\mathbf{m}}(\tilde{\mathbf{m}}, \mathbf{Q})=\tilde{\mathbf{m}}^{\top} \mathbf{Q}^{-1} \tilde{\mathbf{m}}$.

For notational clarity, from this point on we omit the time argument. We note that

$\lambda_{\min }\left(\mathbf{Q}^{-1}\right)\|\tilde{\mathbf{m}}\|^{2} \leq V_{\mathbf{m}}(\tilde{\mathbf{m}}, \mathbf{Q}) \leq \lambda_{\max }\left(\mathbf{Q}^{-1}\right)\|\tilde{\mathbf{m}}\|^{2}$,

where $\lambda_{\min }\left(\mathbf{Q}^{-1}\right)$ and $\lambda_{\max }\left(\mathbf{Q}^{-1}\right)$ are the smallest and largest eigenvalue of $\mathbf{Q}^{-1}$, respectively. For further details on $\mathbf{Q}^{-1}$, the reader is referred to Haring (2016, Chapter 2). From the observer in (33) and (58) we obtain the time derivative of $V_{\mathbf{m}}$ as

$$
\begin{aligned}
\dot{V}_{\mathbf{m}}= & 2 \tilde{\mathbf{m}}^{\top} \mathbf{Q}^{-1} \dot{\tilde{\mathbf{m}}}-\tilde{\mathbf{m}}^{\top} \mathbf{Q}^{-1} \dot{\mathbf{Q}} \mathbf{Q}^{-1} \tilde{\mathbf{m}}, \\
= & -\eta_{\mathbf{m}} \tilde{\mathbf{m}}^{\top} \mathbf{Q}^{-1} \tilde{\mathbf{m}}-\eta_{\mathbf{m}} \tilde{\mathbf{m}}^{\top}\left(\mathbf{C}^{\top} \mathbf{C}+\sigma_{r} \mathbf{D}^{\top} \mathbf{D}\right) \tilde{\mathbf{m}} \\
& +2 \alpha_{\omega}^{2} \tilde{\mathbf{m}}^{\top} \mathbf{Q}^{-1} \mathbf{B}(\hat{\mathbf{s}}-\mathbf{s})-2 \eta_{\mathbf{m}} \sigma_{r} \alpha_{\omega} \tilde{\mathbf{m}}^{\top} \mathbf{D}^{\top} \frac{d F_{\mathbf{w}}}{d \mathbf{u}^{\top}}(\hat{\mathbf{u}}) \\
& -2 \eta_{\mathbf{m}} \tilde{\mathbf{m}}^{\top} \mathbf{C}^{\top}\left(\alpha_{\omega}^{2}(\hat{v}-v)-r-d\right),
\end{aligned}
$$

where we have used the fact that $\mathbf{Q}^{-1}$ is real and symmetric, i.e., $\mathbf{Q}^{-1}=\mathbf{Q}^{-T}$, and, given $\mathbf{A}$ in (31), that $\tilde{\mathbf{m}}^{\top}\left(\mathbf{Q}^{-1} \mathbf{A}-\mathbf{A}^{\top} \mathbf{Q}^{-1}\right)$ $\tilde{\mathbf{m}}=0$. Furthermore, given $\mathbf{C}$ in (31) and $\mathbf{D}=\left[\begin{array}{ll}\mathbf{0}^{n_{\mathbf{u}} \times 1} & \mathbf{I}^{n_{\mathbf{u}} \times n_{\mathbf{u}}}\end{array}\right]$, using the fact that $-\tilde{\mathbf{m}}^{\top} \mathbf{C}^{\top} \mathbf{C} \tilde{\mathbf{m}}=-\left\|\tilde{\mathbf{m}}^{\top} \mathbf{C}^{\top} \mathbf{C} \tilde{\mathbf{m}}\right\|=-\|\mathbf{C} \tilde{\mathbf{m}}\|^{2}$ and $\left\|\tilde{\mathbf{m}}^{\top} \mathbf{C}^{\top}\right\|=\|\mathbf{C} \tilde{\mathbf{m}}\|,(\mathrm{C} .1)$, and Young's inequality, we obtain

$$
\begin{aligned}
\dot{V}_{\mathbf{m}} \leq & -\frac{1}{2} \eta_{\mathbf{m}} V_{\mathbf{m}}(\tilde{\mathbf{m}}, \mathbf{Q})+\eta_{\mathbf{m}} \sigma_{r} \alpha_{\omega}^{2}\left\|\frac{d F_{\mathbf{w}}}{d \mathbf{u}^{\top}}(\hat{\mathbf{u}})\right\|^{2} \\
& +2 \frac{\alpha_{\omega}^{4}}{\eta_{\mathbf{m}}}\left\|\mathbf{Q}^{-1}\right\|\|\mathbf{B}\|^{2}\|\hat{\mathbf{s}}-\mathbf{s}\|^{2} \\
& +3 \eta_{\mathbf{m}} \alpha_{\omega}^{4}|\hat{v}-v|^{2}+3 \eta_{\mathbf{m}}|r|^{2}+3 \eta_{\mathbf{m}}|d|^{2} .
\end{aligned}
$$

From (32), (34), the bound $\|\hat{\mathbf{H}}(t, \hat{\mathbf{u}}(t))\| \leq L_{\mathbf{H}}$, the definition of $\boldsymbol{\omega}$ in (24), which implies that there exists a constant $L_{\omega 2} \in \mathbb{R}_{>0}$ such that $\|\boldsymbol{\omega}\| \leq L_{\omega 2}$, and the bound in (22) we obtain

$\|\hat{\mathbf{s}}-\mathbf{s}\| \leq \frac{1}{\alpha_{\omega}}\left(L_{\mathbf{H}}+L_{F 2}\right)\|\dot{\hat{\mathbf{u}}}\|,|\hat{v}-v| \leq \frac{1}{2}\left(L_{\mathbf{H}}+L_{F 2}\right) L_{\omega 2}^{2}$.

From (32) and the bounds in (12), it follows that

$$
|r|=\left\|\int_{0}^{1} \frac{d k}{d \mathbf{z}}\left(\sigma \tilde{\mathbf{z}}+\overline{\mathbf{z}}_{\mathbf{w}}\right) d \sigma \tilde{\mathbf{z}}\right\| \leq L_{k}\|\tilde{\mathbf{z}}\| .
$$

From (B.7) and by using Young's inequality, it follows that $\| \mathbf{u}-$ $\mathbf{u}_{\mathbf{w}}^{*}\left\|^{2} \leq 2\right\| \tilde{\mathbf{u}} \|^{2}+2 \alpha_{\omega}^{2} L_{\omega 2}^{2}$. From this, (27), the bounds in (12), and
Assumption 11, we obtain

$$
|d| \leq L_{k} \alpha_{\mathbf{z}}\left(\delta_{\mathbf{z} 1}+2 \delta_{\mathbf{z} 2}\|\tilde{\mathbf{u}}\|^{2}+2 \delta_{\mathbf{z} 2} \alpha_{\omega}^{2} L_{\omega 2}^{2}\right) .
$$

From the coordinate transformation in (37) and the bound in (22), we obtain

$$
\begin{aligned}
\left\|\frac{d F_{\mathbf{w}}}{d \mathbf{u}}(\hat{\mathbf{u}})\right\| & \leq \int_{0}^{1}\left\|\frac{d^{2} F_{\mathbf{w}}}{d \mathbf{u} d \mathbf{u}^{\top}}\left(\sigma \tilde{\mathbf{u}}+\mathbf{u}_{\mathbf{w}}^{*}\right)\right\| d \sigma\|\tilde{\mathbf{u}}\| \\
& \leq L_{F 2}\|\tilde{\mathbf{u}}\| .
\end{aligned}
$$

By combining (C.4)-(C.8) and since we have from (31) that $\|\mathbf{B}\|=$ 1 , we obtain

$$
\begin{aligned}
\dot{V}_{\mathbf{m}} \leq & -\frac{1}{2} \eta_{\mathbf{m}} V_{\mathbf{m}}(\tilde{\mathbf{m}}, \mathbf{Q})+\eta_{\mathbf{m}} \sigma_{r} \alpha_{\omega}^{2} L_{F 2}^{2}\|\tilde{\mathbf{u}}\|^{2} \\
& +2 \frac{\alpha_{\omega}^{2}}{\eta_{\mathbf{m}}}\left(L_{\mathbf{H}}+L_{F 2}\right)^{2}\left\|\mathbf{Q}^{-1}\right\|\|\mid \hat{\hat{\mathbf{u}}}\|^{2}+3 \eta_{\mathbf{m}} L_{k}^{2}\|\tilde{\mathbf{z}}\|^{2} \\
& +9 \eta_{\mathbf{m}} L_{k}^{2} \alpha_{\mathbf{z}}^{2}\left(\delta_{\mathbf{z} 1}^{2}+4 \delta_{\mathbf{z} 2}^{2}\|\tilde{\mathbf{u}}\|^{4}+4 \delta_{\mathbf{z} 2}^{2} \alpha_{\omega}^{4} L_{\omega 2}^{4}\right) \\
& +\frac{3}{4} \eta_{\mathbf{m}} \alpha_{\omega}^{4}\left(L_{\mathbf{H}}+L_{F 2}\right)^{2} L_{\omega 2}^{4} .
\end{aligned}
$$

From Lemmas 19 and 20 we have that, for any finite time $t_{1} \geq$ 0 , the solutions $\tilde{\mathbf{u}}$ and $\tilde{\mathbf{z}}$ are bounded for all $0 \leq t \leq t_{1}$. Moreover, from Lemma 18 we have that $\mathbf{Q}^{-1}$ is positive definite and bounded for all $0 \leq t \leq t_{1}$. From these facts and $\|\dot{\hat{\mathbf{u}}}\| \leq \eta_{\mathbf{u}}$, which follows from (35), we obtain that the right-hand side of (C.9) is bounded for all $0 \leq t \leq t_{1}$. Therefore, $V_{\mathbf{m}}(\tilde{\mathbf{m}}(t), \mathbf{Q}(t))$ will be bounded for all $0 \leq t \leq t_{1}$, and $\mathbf{Q}^{-1}$ is positive definite and bounded for all $0 \leq t \leq t_{1}$ as well, it follows from (C.2) that the solutions $\tilde{\mathbf{m}}$ are bounded for all $0 \leq t \leq t_{1}$.

Let us define $t_{1} \geq 0$ such that from Lemmas 17 and 18 we have that $\|\tilde{\mathbf{x}}(t)\| \leq \alpha_{\omega} \eta_{\omega} c_{\mathbf{x} 2}$ and $\|\tilde{\mathbf{Q}}(t)\| \leq \frac{1}{8}$, for all $t \geq t_{1}$. From the proof of Lemma 2.11 in Haring (2016, Chapter 2), we have that $\frac{1}{4} \mathbf{I} \preceq \mathbf{Q}^{-1} \preceq \frac{5}{4} \mathbf{I}$ for all $t \geq t_{1}$, all $\eta_{\mathbf{m}} \leq \eta_{\omega} \epsilon_{3}$ and all $\sigma_{r} \leq \epsilon_{6}$, with $\epsilon_{3}$ and $\epsilon_{6}$ sufficiently small. Moreover, it follows that $\frac{1}{4}\|\tilde{\mathbf{m}}\|^{2} \leq V_{\mathbf{m}}(\tilde{\mathbf{m}}, \mathbf{Q}) \leq \frac{5}{4}\|\tilde{\mathbf{m}}\|^{2}$, for all $t \geq t_{1}$, and $\left\|\mathbf{Q}^{-1}\right\| \leq \frac{5}{4}$ for all $t \geq t_{1}$. From (25), (35), (37), $\|\mathbf{D}\|=1$, and the bound in (22), it follows that $\|\dot{\hat{\mathbf{u}}}\|^{2} \leq 8 \lambda_{\mathbf{u}}^{2} V_{\mathbf{m}}(\tilde{\mathbf{m}}, \mathbf{Q})+2 \alpha_{\omega}^{2} \lambda_{\mathbf{u}}^{2} L_{\mathrm{F} 2}^{2}\|\tilde{\mathbf{u}}\|^{2}$, for all $t_{1} \geq 0$. From this, and taking $\epsilon_{4}$ in Theorem 14 sufficiently small, we obtain from (C.9) that

$$
\begin{aligned}
\dot{V}_{\mathbf{m}} \leq & -\frac{1}{4} \eta_{\mathbf{m}} V_{\mathbf{m}}(\tilde{\mathbf{m}}, \mathbf{Q})+\eta_{\mathbf{m}} \sigma_{r} \alpha_{\omega}^{2} L_{F 2}^{2}\|\tilde{\mathbf{u}}\|^{2} \\
& +5 \frac{\alpha_{\omega}^{4} \lambda_{\mathbf{u}}^{2}}{\eta_{\mathbf{m}}}\left(L_{\mathbf{H}}+L_{F 2}\right)^{2} L_{F 2}^{2}\|\tilde{\mathbf{u}}\|^{2} \\
& +\frac{3}{4} \eta_{\mathbf{m}} \alpha_{\omega}^{4}\left(L_{\mathbf{H}}+L_{F 2}\right)^{2} L_{\omega 2}^{4}+3 \eta_{\mathbf{m}} L_{k}^{2}\|\tilde{\mathbf{z}}\|^{2} \\
& +9 \eta_{\mathbf{m}} L_{k}^{2} \alpha_{\mathbf{z}}^{2}\left(\delta_{\mathbf{z} 1}^{2}+4 \delta_{\mathbf{z} 2}^{2}\|\tilde{\mathbf{u}}\|^{4}+4 \delta_{\mathbf{z} 2}^{2} \alpha_{\omega}^{4} L_{\omega 2}^{4}\right),
\end{aligned}
$$

for all $t \geq t_{1}$, and all $\alpha_{\omega} \lambda_{\mathbf{u}} \leq \eta_{\mathbf{m}} \epsilon_{4}$. From the comparison lemma and the inequality in (C.10), and using (C.2), we obtain

$$
\begin{aligned}
& \sup _{t \geq t_{1}}\|\tilde{\mathbf{m}}(t)\| \leq \sqrt{32} \sup _{t \geq t_{1}} \max \left\{\sqrt{\frac{5}{4}}\left\|\tilde{\mathbf{m}}\left(t_{1}\right)\right\|,\right. \\
& \quad 2 \sqrt{5} \frac{\alpha_{\omega}^{2} \lambda_{\mathbf{u}}}{\eta_{\mathbf{m}}}\left(L_{\mathbf{H}}+L_{F 2}\right) L_{F 2}\|\tilde{\mathbf{u}}(t)\|, 2 \sqrt{3} L_{k}\|\tilde{\mathbf{z}}(t)\|, \\
& \sqrt{3} \alpha_{\omega}^{2}\left(L_{\mathbf{H}}+L_{F 2}\right) L_{\omega 2}^{2}, 2 \sqrt{\sigma_{r}} \alpha_{\omega} L_{F 2}\|\tilde{\mathbf{u}}(t)\|, \\
& \left.\quad 12 \alpha_{\mathbf{z}} \delta_{\mathbf{z} 2} L_{k}\|\tilde{\mathbf{u}}(t)\|^{2}, 12 \alpha_{\mathbf{z}} \delta_{\mathbf{z} 2} \alpha_{\omega}^{2} L_{k} L_{\omega 2}^{2}, 6 \alpha_{\mathbf{z}} \delta_{\mathbf{z} 1} L_{k}\right\} \\
& \limsup _{t \rightarrow \infty}\|\tilde{\mathbf{m}}(t)\| \leq 2 \sqrt{6} \limsup _{t \rightarrow \infty}\{ \\
& 2 \sqrt{5} \frac{\alpha_{\omega}^{2} \lambda_{\mathbf{u}}}{\eta_{\mathbf{m}}}\left(L_{\mathbf{H}}+L_{F 2}\right) L_{F 2}\|\tilde{\mathbf{u}}(t)\|, 2 \sqrt{3} L_{k}\|\tilde{\mathbf{z}}(t)\|, \\
& \sqrt{3} \alpha_{\omega}^{2}\left(L_{\mathbf{H}}+L_{F 2}\right) L_{\omega 2}^{2}, 2 \sqrt{\sigma_{r}} \alpha_{\omega} L_{F 2}\|\tilde{\mathbf{u}}(t)\|, \\
& \left.12 \alpha_{\mathbf{z}} \delta_{\mathbf{z} 2} L_{k}\|\tilde{\mathbf{u}}(t)\|^{2}, 12 \alpha_{\mathbf{z}} \delta_{\mathbf{z} 2} \alpha_{\omega}^{2} L_{k} L_{\omega 2}^{2}, 6 \alpha_{\mathbf{z}} \delta_{\mathbf{z} 1} L_{k}\right\} .
\end{aligned}
$$

for all $t \geq t_{1}$, all $\eta_{\mathbf{m}} \leq \eta_{\omega} \epsilon_{3}$, all $\alpha_{\omega} \lambda_{\mathbf{u}} \leq \eta_{\mathbf{m}} \epsilon_{4}$, and all $\sigma_{r} \leq \epsilon_{6}$. The bounds in (59) and (60) of Lemma 22 follow from (C.11) and (C.12), respectively, which completes the proof of Lemma 22. 


\section{References}

Antonello, R., Oboe, R., Prandi, L., \& Biganzoli, F. (2009). Automatic mode matching in MEMS vibrating gyroscopes using extremum-seeking control. IEEE Transactions on Industrial Electronics, 56(10), 3880-3891.

Bolder, J. J., Witvoet, G., de Baar, M. R., van de Wouw, N., Haring, M. A. M., Westerhof, E., et al. (2012). Robust sawtooth period control based on adaptive online optimization. Nuclear Fusion, 52(7), 074006.

Cao, Z., Dürr, H-B., Ebenbauer, C., Allgöwer, F., \& Gao, F. (2017). Iterative learning and extremum seeking for repetitive time-varying mappings. IEEE Transactions on Automatic Control, 62(7), 3339-3353.

Dürr, H.-B., Krstić, M., Scheinker, A., \& Ebenbauer, C. (2017). Extremum seeking for dynamic maps using Lie brackets and singular perturbations. Automatica, 83, 91-99.

Freudenberg, J., Middleton, R., \& Stefanpoulou, A. (2000). A survey of inherent design limitations. In Proc. am. control. conf., Vol. 5 (pp. 2987-3001).

Fu, L., \& Özgüner, Ü. (2011). Extremum seeking with sliding mode gradient estimation and asymptotic regulation for a class of nonlinear systems. Automatica, 47(12), 2595-2603.

Gelbert, G., Moeck, J. P., Paschereit, C. O., \& King, R. (2012). Advanced algorithms for gradient estimation in one- and two-parameter extremum seeking controllers. Journal of Process Control, 22(4), 700-709.

Grushkovskaya, V., Dürr, H.-B., Ebenbauer, C., \& Zuyev, A. (2017). Extremum seeking for time-varying functions using Lie bracket approximations. IFAC-PapersOnLine, 50(1), 5522-5528, 20th IFAC World Congress.

Guay, M., \& Dochain, D. (2015). A time-varying extremum-seeking contro approach. Automatica, 51, 356-363.

Guay, M., Dochain, D., Perrier, M., \& Hudon, N. (2007). Flatness-based extremumseeking control over periodic orbits. IEEE Transactions on Automatic Control, 52(10), 2005-2012

Haring, M. A. M. (2016). Extremum-seeking control: convergence improvements and asymptotic stability ( $\mathrm{PhD}$ thesis), Trondheim: Dept. of Engineering Cybernetics, Norwegian University of Science and Technology.

Haring, M., \& Johansen, T. A. (2018). On the accuracy of gradient estimation in extremum-seeking control using small perturbations. Automatica, 95, 23-32.

Haring, M. A. M., van de Wouw, N., \& Nešić, D. (2013). Extremum-seeking control for nonlinear systems with periodic steady-state outputs. Automatica, 49(6), 1883-1891.

Hazeleger, L., Haring, M. A. M., \& van de Wouw, N. (2018). Extremum-seeking control for steady-state performance optimization of nonlinear plants with time-varying steady-state outputs. In Proc. am. control conf. (pp. 2990-2995).

Heertjes, M. F., \& Nijmeijer, H. (2012). Self-tuning of a switching controller for scanning motion systems. Mechatronics, 22(3), 310-319.

Heertjes, M. F., Schuurbiers, X. G. P., \& Nijmeijer, H. (2009). Performanceimproved design of N-PID controlled motion systems with applications to wafer stages. IEEE Transactions on Industrial Electronics, 56(5), 1347-1355

Höffner, K., Hudon, N., \& Guay, M. (2007). On-line feedback control for optima periodic control problems. The Canadian Journal of Chemical Engineering, 85(4), 479-489.

Hunnekens, B. G. B., Di Dino, A., van de Wouw, N., van Dijk, N., \& Nijmeijer, H. (2015). Extremum-seeking control for the adaptive design of variable gain controllers. IEEE Transactions on Control Systems Technology, 23(3), 1041-1051.

Khalil, H. K. (2002). Nonlinear systems. New Jersey: Prentice Hall.

Krstić, M., \& Wang, H.-H. (2000). Stability of extremum seeking feedback for general nonlinear dynamic systems. Automatica, 36(4), 595-601.

Liu, T., Hill, D. J., \& Jiang, Z.-P. (2011). Lyapunov formulation of ISS cyclicsmall-gain in continuous-time dynamical networks. Automatica, 47(9), 2088-2093.

Nešić, D., Tan, Y., Moase, W. H., \& Manzie, C. (2010). A unifying approach to extremum seeking: Adaptive schemes based on estimation of derivatives. In Proc IEEE conf decis control (pp. 4625-4630).

Pavlov, A. V., Hunnekens, B. G. B., van de Wouw, N., \& Nijmeijer, H. (2013). Steady-state performance optimization for nonlinear control systems of Lure type. Automatica, 49(7), 2087-2097.

Pavlov, A. V., \& van de Wouw, N. (2016). Convergent systems: Nonlinear simplicity. In Lecture notes in control and information sciences: vol. 470 Nonlinear systems, techniques for dynamical analysis and control (pp. 51-77). Springer.

Pavlov, A. V., van de Wouw, N., \& Nijmeijer, H. (2006). Uniform output regulation of nonlinear systems: a convergence dynamics approach. Boston: Birkhäuser.

Rušiti, D., Evangelisti, G., Oliveira, T. R., Gerdts, M., \& Krstić, M. (2019). Stochastic extremum seeking for dynamic maps with delays. IEEE Control System Letters, 3(1), 61-66.

Sahneh, F. D., Hu, G., \& Xie, L. (2012). Extremum seeking control for systems with time-varying extremum. In Proc. 31st Chinese control conf. (pp. 225-231).

Scheinker, A., \& Krstić, M. (2013). Minimum-seeking for CLFs: Universal semiglobally stabilizing feedback under unknown control directions. IEEE Transactions on Automatic Control, 58(5), 1107-1122.
Scheinker, A., \& Scheinker, D. (2016). Bounded extremum seeking with discontinuous dithers. Automatica, 69, 250-257.

Stanković, M. S., \& Stipanović, D. M. (2010). Extremum seeking under stochastic noise and applications to mobile sensors. Automatica, 46(8), 1243-1251.

Tan, Y., Moase, W. H., Manzie, C., Nešić, D., \& Mareels, I. M. Y. (2010). Extremum seeking from 1922 to 2010. In Proc. 29th Chinese control conf. (pp. 14-26).

Tan, Y., Nešić, D., \& Mareels, I. (2006). On non-local stability properties of extremum seeking control. Automatica, 42(6), 889-903.

Teel, A. R., \& Popović, D. (2001). Solving smooth and nonsmooth multivariable extremum seeking problems by the methods of nonlinear programming. In Proc. am. control conf., Vol. 3 (pp. 2394-2399).

van Loon, S. J. L. M., Hunnekens, B. G. B., Heemels, W. P. M. H., van de Wouw, N., \& Nijmeijer, H. (2016). Split-path nonlinear integral control for transient performance improvement. Automatica, 66, 262-270.

Wang, H.-H., \& Krstić, M. (2000). Extremum seeking for limit cycle minimization. IEEE Transactions on Automatic Control, 45(12), 2432-2436.

Zhang, C., \& Ordóñez, R. (2009). Robust and adaptive design of numerical optimization-based extremum seeking control. Automatica, 45(3), 634-646.

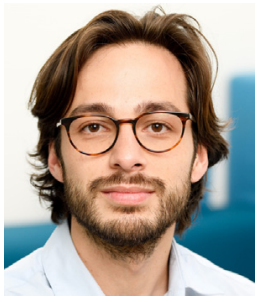

Leroy Hazeleger received his B.Sc. degree in Mechanical Engineering at the Eindhoven University of Technology, The Netherlands, in 2012, and his M.Sc. degree in Systems and Control within the Department of Mechanical Engineering at the Eindhoven University of Technology, The Netherlands, in 2015. In 2016, he started his Ph.D. research within the Dynamics and Control Group at the Eindhoven University of Technology, The Netherlands. His current research interests include nonlinear control systems, datadriven optimization, and extremum-seeking control, with applications to high-performance mechatronic systems.

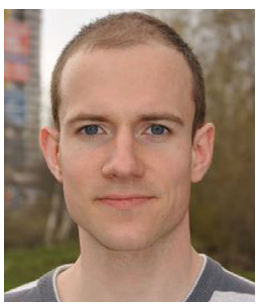

Mark Haring received his B.Sc. degree and M.Sc. degree in mechanical engineering from Eindhoven University of Technology, Eindhoven, The Netherlands, in 2008 and 2011, respectively. In 2016, he obtained his Ph.D. degree in engineering cybernetics at NTNU, the Norwegian University of Science and Technology, Trondheim, Norway. He is currently working as a research scientist at SINTEF Digital, Mathematics and Cybernetics, Trondheim, Norway. His research interests include automatic control, adaptive control, and estimation.

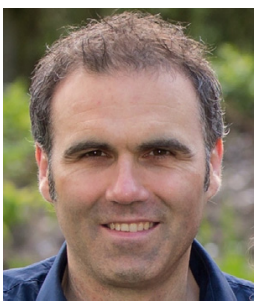

Nathan van de Wouw (born, 1970) obtained his M.Sc.degree (with honors) and Ph.D.-degree in Mechanical Engineering from the Eindhoven University of Technology, Eindhoven, the Netherlands, in 1994 and 1999, respectively. He currently holds a full professor position at the Mechanical Engineering Department of the Eindhoven University of Technology, the Netherlands. Nathan van de Wouw also holds an adjunct full professor position at the University of Minnesota, U.S.A. In 2000, Nathan van de Wouw has been working at Philips Applied Technologies, Eindhoven, The Netherlands, and, in 2001, he has been working at the Netherlands Organisation for Applied Scientific Research (TNO), Delft, The Netherlands. He has held positions as a visiting professor at the University of California Santa Barbara, U.S.A., in 2006/2007, at the University of Melbourne, Australia, in 2009/2010 and at the University of Minnesota, U.S.A., in 2012 and 2013. He has held a (part-time) full professor position the Delft University of Technology, the Netherlands, from 2015-2019. He has published the books 'Uniform Output Regulation of Nonlinear Systems: A convergent Dynamics Approach' with A.V. Pavlov and H. Nijmeijer (Birkhauser, 2005) and 'Stability and Convergence of Mechanical Systems with Unilateral Constraints' with R.I. Leine (Springer-Verlag, 2008). He currently is an Associate Editor for the journals "Automatica" and "IEEE Transactions on Control Systems Technology".

In 2015, he received the IEEE Control Systems Technology Award "For the development and application of variable-gain control techniques for high performance motion systems". His current research interests are the modeling, model reduction, analysis and control of nonlinear/hybrid and delay systems, with applications to vehicular platooning, high-tech systems, resource exploration, smart energy systems and networked control systems. 